\title{
Genetic Polymorphisms of Pesticide-Metabolizing Enzymes and Transporters in Agricultural Workers and Thyroid Hormone Levels
}

Jintana Sirivarasai ${ }^{1}$

Suwanee Chanprasertyothin ${ }^{2}$

Pornpimol Kongtip (iD) ${ }^{3}$

Susan Woskie ${ }^{4}$

'Faculty of Medicine Ramathibodi Hospital, Mahidol University, Bangkok, Thailand; ${ }^{2}$ Research and Innovation Center, Faculty of Medicine Ramathibodi Hospital, Mahidol University, Bangkok, Thailand; ${ }^{3}$ Department of Occupational Health and Safety, Faculty of Public Health, Mahidol University, Bangkok, Thailand; ${ }^{4}$ Department of Public Health, University of Massachusetts Lowell, Lowell, MA, USA
Correspondence: Jintana Sirivarasai Faculty of Medicine Ramathibodi Hospital, Mahidol University, 270 Rama 6 Road,

Ratchatavee District, Bangkok, I0400,

Thailand

Tel +66 2-20I-I483

Fax+66 2-20I-2625

Email jintana.sir@mahidol.ac.th
Purpose: Chronic exposure to pesticides has been associated with thyroid dysfunction owing to their endocrine disruption ability. Genetic variations in genes encoding phase I and II enzymes and phase III transporters are partly responsible for individual responses to chemical pesticides. This study investigated the association between variations in genes involved in pesticide metabolism and altered thyroid hormone concentrations.

Methods: We assessed thyroid-stimulating hormone (TSH), free triiodothyronine (FT3), and free thyroxine (FT4) in organic agriculture workers $(n=216)$ and workers who used chemical pesticides $(n=229)$. A questionnaire was used to collect sociodemographic, pesticide exposure, and health status data. Blood samples were analyzed for TSH, FT3, and FT4. Genomic DNA was extracted and genotyped using the TaqMan real-time PCR genotyping assay and restriction fragment length polymorphism method for 15 metabolically related genes.

Results: Significant differences in the TSH $(1.58$ vs $1.12 \mu \mathrm{IU} / \mathrm{mL})$ and FT3 (0.34 vs 0.31 $\mathrm{ng} / \mathrm{dL}$ ) concentrations between the chemical and organic worker groups were observed. The frequencies of all single nucleotide polymorphisms were in Hardy-Weinberg equilibrium and were mostly consistent with Asian populations. The findings showed the association between SNPs of enzymes and transporters and TSH, FT3, and FT4. The odd ratio and adjusted odd ratio (with sex, age, smoking status, alcohol consumption and exposure parameters) for subclinical thyroid disease by the variant alleles CYP1A1 rs1048943, CYP2B6 rs2279343, CYP2C19 rs4244285, NAT2 rs1799931, and PON1 rs662 in the chemical workers compared with the organic workers were found $(P$ values $<0.05)$.

Conclusion: This is the first study to assess gene-environment interactions in Thai agricultural workers by investigating disruptions of the hypothalamic-pituitary-thyroid axis. The investigated SNP profiles revealed several gene-thyroid hormone associations in which even low levels of pesticide exposure could disturb thyroid homeostasis. These findings provide a foundation for planning future studies investigating associations between complex diseases and occupational pesticide exposure.

Keywords: occupational pesticide exposure, enzyme phase I and II, transporters, genotype, thyroid-stimulating hormone

\section{Introduction}

Pesticides are widely used in many countries, including Thailand. Thailand is a major agricultural exporter in Southeast Asia, and there has been a considerable increase in pesticide importation in recent years. Herbicides constitute the largest proportion of imported pesticides, followed by insecticides, fungicides, and other pesticide 
groups. ${ }^{1}$ The most common imported herbicides are glyphosate, paraquat, and 2,4-D-dimethylammonium; the most common imported insecticides are chlorpyrifos (an organophosphorus compound), cypermethrin (a synthetic pyrethroid), carbaryl (a carbamate), and carbosulfan (also a carbamate). ${ }^{1}$ Previous studies have indicated similar trends in pesticide use by Thai agricultural workers. A study of 424 pesticide-exposed workers from farms producing rice, vegetables, and/or flowers found herbicide, insecticide, and fungicide usage levels of 46.9-64.9\%, $22.6-26.3 \%$, and $10.6-25.2 \%$, respectively. ${ }^{2}$ One study that investigated pesticide exposure and health symptoms found that among the included 182 rice farmers, $84.6 \%$ used insecticides, $63.2 \%$ used herbicides, and $17.1 \%$ used fungicides. ${ }^{3}$ Exposure to various subgroups of these pesticides can result in acute and chronic adverse health effects.

Human susceptibility to pesticide toxicity may depend on age, sex, exposure factors (chemical properties, route, dose, and duration), health status (lifestyle, medical history), nutritional status, and genetic factors. Genetic polymorphisms are one determinant of pesticide-induced adverse health effects, especially polymorphisms of genes related to pesticide metabolism. ${ }^{4}$ Few studies have focused on genetic variation in the different steps of pesticide metabolism, such as variation in the phase I and II enzymes and phase III transporters. The most common pathways in phase I metabolism are related to oxidation by cytochrome $\mathrm{P} 450$, as well as reduction and hydrolysis reactions. The cytochrome P450 (CYP) superfamily is the main enzyme group involved in pesticide biotransformation; members include CYP1A1, CYP1A2, CYP2B6, CYP2D6, CYP2C9, CYP2C19, CYP3A4, and CYP3A5. This family of enzymes shows significant variability in catalytic activity. Phase II enzymes play a role in glucuronidation, acetylation, sulfation, and glutathione conjugation and include glutathione S-transferase (GST), UDPglucuronosyltransferase, N-acetyltransferase (NAT), and sulfotransferase. Additionally, there are two main superfamilies of phase III transporters, ATP-binding cassette (ABC) transporters and solute carrier transporters, which actively take up or efflux parent compounds and toxic intermediates. ${ }^{4}$ Our previous work studied polymorphisms of the paraoxonase 1 (PON1) gene in the coding and promoter regions $(\mathrm{Q} 192 \mathrm{R}$, L55M, and T-108C) and enzyme activities (cholinesterase, paraoxonase, arylesterase, and diazonase) in an organophosphate (OP)-exposed population. The findings supported the role of PON1 in inhibiting cholinesterase. ${ }^{5}$

An important issue related to off-target effects of pesticides is endocrine dysfunction, which has been noted for a variety of pesticide classes; such chemicals are termed endocrine-disrupting chemicals (EDCs). EDCs can impact human health by modifying various hormone receptors and influencing their binding ability. EDCs can also alter the synthesis, metabolism, and clearance of endogenous hormones, thereby disrupting hormone levels. ${ }^{6}$ There is increasing evidence of pesticides acting as EDCs to disrupt thyroid function. One study found that chlorpyrifos (CPF) induced significant changes in the triiodothyronine (T3) and thyroxine (T4) concentrations of CPF-exposed adult Wistar rats compared with a control group $(-28 \%, P<0.05$ and $-36.06 \%, P<0.01$, respectively) and increased oxidative damage in the thyroid gland. However, animals exposed to both CPF and medicinal plants (antioxidant and antiinflammatory herbs) showed significantly $(P<0.05)$ higher serum T3 and T4 concentrations compared with those exposed to $\mathrm{CPF}$ alone. ${ }^{7}$ A possible mechanism of the adverse effect of CPF may involve the oxidative stress arising from the parent compound's biotransformation into toxic metabolites, with the concomitant generation of reactive oxygen species (ROS). ${ }^{8}$ However, a causal relationship between occupational pesticide exposure-induced changes in the hypothalamic-pituitary-thyroid (HPT) axis and genetic variations of the enzymes and transporters involved in pesticide metabolism has not been established. To our knowledge, no studies have examined the association between circulating thyroid hormone concentrations and the variability of genes involved in the metabolic phases of pesticide biotransformation. Therefore, this study investigated genetic variations in phase I and II enzymes and phase III transporters and their effects on thyroid hormone concentrations among agricultural workers who are exposed to pesticides and those who work on organic farms.

\section{Materials and Methods Study Population}

This work is a part of a longitudinal study which will evaluate the sub-chronic effects of repeated exposure to different types of pesticides on thyroid hormone levels. This study was conducted in an agricultural area that is representative of the crops grown in Thailand and the pesticide usage in the country. Participants were recruited from February-April 2016. All participants underwent a physical examination during which anthropometric and blood pressure measurements were taken by personnel who had been trained in these standard procedures. There were two study groups: 1) The chemical worker group $(n=216)$ included 
those who currently sprayed pesticides at rice and vegetable farms in Phitsanulok Province or at a sugarcane farm in Nakhon Sawan Province. 2) The organic worker group $(n=229)$ included those who currently worked under certified organic practices (with history of no use any chemical pesticides at least five years under inspection and certification services under the accreditation system by the Ministry of Agriculture and Cooperatives, Thailand) at rice, fruit, and vegetable farms in Yasothon Province. The inclusion criteria were individuals over 18 years of age who did have not a diagnosis of, or prior medical treatment for thyroid disease, diabetes mellitus, hypertension, cardiovascular disease, or endocrine diseases. The questionnaires related to pesticides exposure comprised screening questions, worker characteristics, self-reported health problems, agricultural activities, work time in agricultural field (hour/week), duration time for pesticide use, and current/history of pesticide use. Moreover, at the time the chemical workers sprayed pesticides, they had to fill details in the record form that included information about the type of pesticide sprayed, quantity of pesticide used, area sprayed, and any health symptoms after spraying.

In this study, the chemical workers reported using three main groups of pesticides: herbicides (eg, paraquat, glyphosate, and propanil), insecticides (eg, chlorpyrifos, methyl parathion, carbofuran, and cypermethrin), and fungicides (eg, mancozeb, propiconazole, and difenoconazole). These chemicals were sprayed on rice, vegetable, and/or sugarcane fields (Figure 1). The mean time of using agricultural pesticides was $25.3 \pm 14.3$ years. The self-reported pesticide use showed that $35.4 \%$ of workers had worked with these chemicals for more than 30 years (range 4-51 years).

The study was performed in accordance with the principles of the Declaration of Helsinki and was approved by the ethics committee of the Faculty of Medicine Ramathibodi Hospital, Mahidol University (MU-RA 2019/139). Written informed consent was obtained from all participants.

\section{Biochemical and Genetic Analysis}

Venous blood samples were collected in the morning after an overnight fast (12 hours), and serum samples were separated and stored at $-80^{\circ} \mathrm{C}$ for subsequent analysis. The plasma blood glucose concentration and lipid profile were determined using an AU5800 (Beckman Coulter, Atlanta, GA, USA) at the local hospital. The concentrations of thyroid hormones (thyroid-stimulating hormone (TSH), free triiodothyronine (FT3), and free thyroxine (FT4)) were determined by a chemiluminescent immunoassay. $^{9-11}$
We selected single nucleotide polymorphisms (SNPs) based on previous studies and the NCBI database. Genomic DNA was extracted using a High Pure PCR Template Preparation Kit (Roche Diagnostics) and was stored at $-20^{\circ} \mathrm{C}$ until genotype analysis. SNPs were determined by TaqMan ${ }^{\circledR}$ assay-based real-time PCR. The following predesigned TaqMan SNP genotyping assays were used (Applied Biosystems, Foster City, CA, USA): C_25624888_50 (CYP1A1, dbSNP: rs1048943), C_15859191_30 (CYP1A2, dbSNP: rs2069514), C_ 8881221_40 (CYP1A2, dbSNP: rs762551), C 26201809 30 (CYP3A5, dbSNP: rs762551), C_7817765_60 (CYP2B6, dbSNP: rs3745274), C_30634238_F0 (CYP2B6, dbSNP: rs2279343), C_25986767_70 (CYP2C19, dbSNP: rs4244285), C_11484460_40 (CYP2D6, dbSNP: rs1065852), C_27102425_50 (CYP2D6, dbSNP: rs16947), C_2431875_10 (CYP2E1, dbSNP: rs3813867), C__8890131_30 (POR1, dbSNP: rs1057868), C_8890129_20 (POR1, dbSNP: rs1135612), C_8684085_20 (NAT2, dbSNP: rs1041983), C_572770_20 (NAT2, dbSNP: rs1799931), C_1204091_10 (NAT2, dbSNP: rs1799930), C 2548962_20 (PON1, dbSNP: rs662), C_11708905_10 (PON1, dbSNP: rs705379), C_2259750_20 (PON1, dbSNP: rs854560), C_ 7586657_20 (ABCB1, dbSNP: rs1045642), C_ 7586662_10 (ABCB1, dbSNP: rs1128503), C_7586662_10 (GSTP1, dbSNP: rs1695). Custom TaqMan SNP genotyping assays were used for C_30957497_10 (GSTM1) and C_30634020_10 (GSTT1). The protocols recommended by the manufacturer were followed.

Genotyping of CYP1A1 (rs4646903), CYP2E1 (rs2031920 and rs6413432), NAT1 (rs15561 and rs1057126) polymorphisms was performed by PCRrestriction fragment length polymorphism (PCR-RFLP) in accordance with previous studies. ${ }^{12-14}$ The temperature was controlled by a thermal cycler (Gene Amp PCR System 9700; Applied Biosystems, Carlsbad, CA, USA). Restriction enzymes were obtained from New England Biolabs (Hitchin, UK) and Fermentas (Vilnius, Lithuania). Primers and restriction enzymes were as in previous studies: for CYP1A1 (rs4646903), F: 5'-CAGTGAAGAGGT GTAGCCGCT-3' and R: 5'-TAGGAGTCTTG TTGTCTCATGCCT-3' and MspI; for CYP2E1 (rs2031920), F: 5'-CCAGTCGAGTCTA-CATTGTCA-3' and R: 5'-TTCATTCTGTCTTCTAACTGG-3' and RsaI 


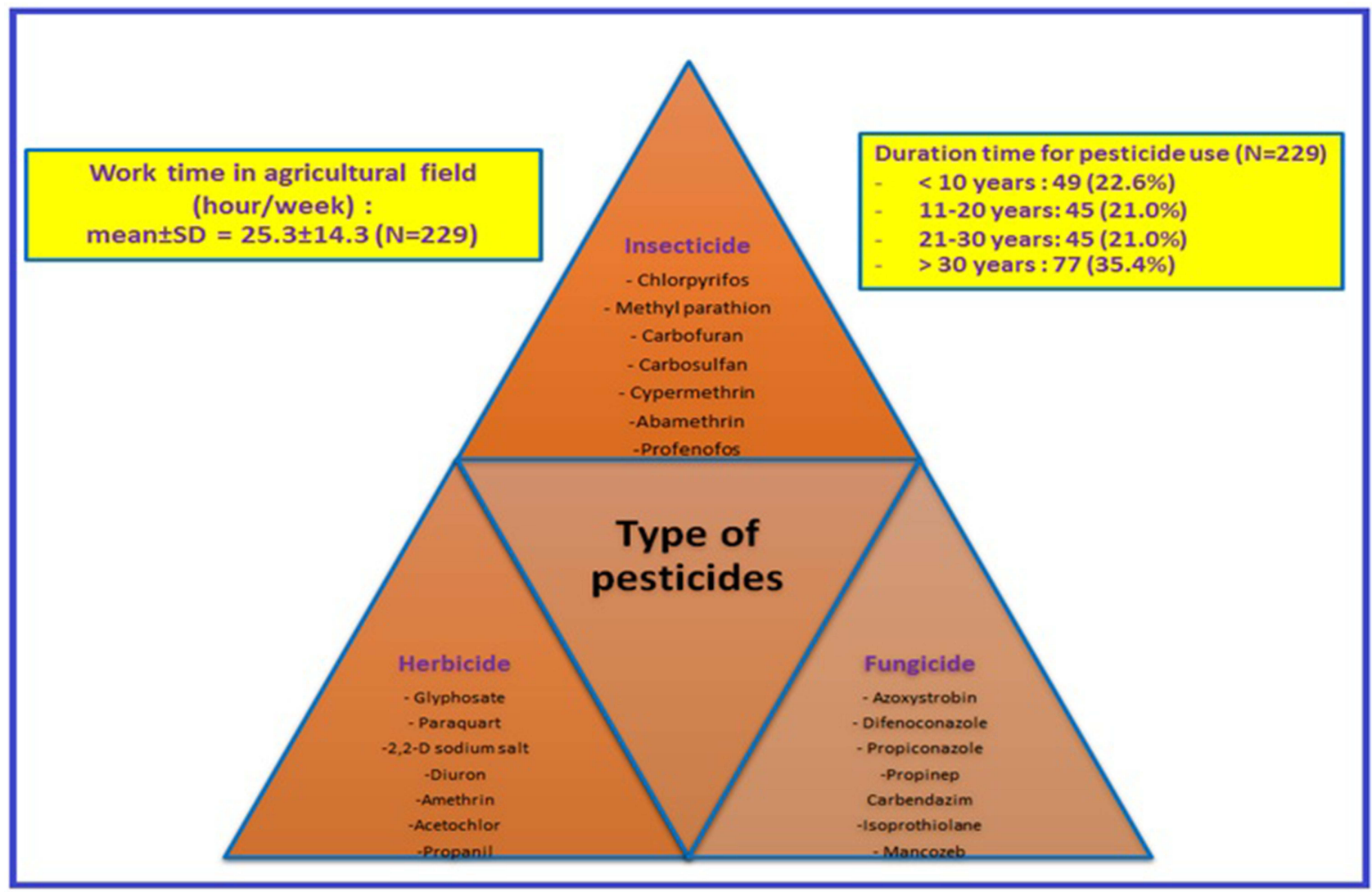

Figure I Pesticide exposure of the chemical workers $(n=216)$.

and PstI; for CYP2E1 (rs6413432), F: 5'CGACATGTGAATGGATCCAGGG-3' and R: 5'-TCGTGA TCGCCTGCCTCA-3' and DraI; ${ }^{12,13}$ and for NAT1 (rs15561 and rs1057126), F: 5'-TTAGGAATTCATGGACATT GAAGCATATCTTGAAAGAAT-3' and R: 5'-GCTTTCT AGCATAAATCACCAA-3' and BanI and AatII. ${ }^{14}$ At least $20 \%$ of the selected PCR-amplified DNA samples were examined by dideoxy DNA sequencing to confirm each polymorphism RFLP result.

\section{Statistical Analysis}

Statistical analyses were performed using SPSS for Windows version 23.0 (IBM, Inc., Armonk, NY, USA). Data are presented as the mean \pm SD for continuous variables, and frequency (\%) for categorical variables. Continuous variables were evaluated using the Kolmogorov-Smirnov test for normality, and skewed data for TSH, FT3, and FT4 were log-transformed Continuous data were compared between the chemical and organic worker genotypes by Student's $t$-test and between two or three genotype groups by Student's $t$-test and one-way analysis of variance. However, in the first step of statistical analysis for thyroid hormone level in both chemical and organic groups, the results showed the absence of sex difference detection as a critical and relevant outcome. Chi-squared analysis was used to determine whether the genotype distribution was in Hardy-Weinberg equilibrium (HWE) and to compare distributions of alleles and genotypes. Normal thyroid function was defined as thyroid hormone concentrations within the normal range. The normal reference ranges of the thyroid hormones in this study were: TSH $(0.45-4.50 \mu \mathrm{IU} / \mathrm{mL})$, FT3 $(0.22-0.42$ $\mathrm{ng} / \mathrm{dL})$, and FT4 (0.78-1.56 ng/dL). ${ }^{15-17}$ Binary logistic regression analysis was performed to identify potential genetic risk factors for thyroid dysfunction, and the odds ratios (ORs) and adjusted ORs (AORs), for sex, age, smoking status, alcohol consumption, and exposure parameters, were calculated. Factors that were found to contribute to the outcome in the univariate analysis at $\mathrm{P}$ value $<0.1$ were included to the multivariate. Multivariate analysis was performed by binary logistic regression analysis, which allows adjust for confounding factors. For each variable, the odds ratio (OR), and 95\% confidence interval (CI) was given. A multivariable model was used to adjust 
for sex, age, smoking status, alcohol consumption, and exposure parameters as possible confounding factors. Parameters associated with thyroid dysfunction were identified at the following altered hormone concentrations: $\mathrm{TSH}<0.45$ or $>4.50 \mu \mathrm{IU} / \mathrm{mL}$; FT3 $<0.22$ or $>0.42 \mathrm{ng} /$ $\mathrm{dL}$; and FT4 $<0.78$ or $>1.56 \mathrm{ng} / \mathrm{dL}$. Subclinical thyroid dysfunction includes subclinical hypo- and hyperthyroidism. These conditions have been defined as follows: high or low serum thyroid stimulating hormone (TSH) levels, normal free T4 and T3 levels and the absence of signs and symptoms of overt thyroid dysfunction. ${ }^{17}$ The findings were considered to be statistically significant at a $P$ value of $<0.05$. Associations among pesticide exposure, polymorphisms, and thyroid hormones were identified, and interactions among these parameters were integrated using STITCH software, a tool used to assess proteinchemical interactions. ${ }^{18}$ Finally, we proposed mechanisms by which pesticide exposure and xenobiotic-metabolizing enzymes and transporters interact to alter thyroid hormone concentrations.

\section{Results}

\section{General Characteristics of the Study Population}

The demographic characteristics of the chemical and organic worker groups are shown in Table 1. The mean age of the organic workers (54.6 \pm 9.3 years) was significantly higher than that of the chemical workers $(51.1 \pm 9.8$ years, $P=0.003$ ). More than $70 \%$ of the chemical workers were men compared with $51.5 \%$ of the organic workers. Some lifestyle factors might increase the risk of thyroid disorders, including smoking and alcohol consumption. The chemical worker group had significantly higher proportions of those who smoked $(26.9 \%)$ and consumed alcohol (63.8\%) compared with the organic worker group ( $16.1 \%$ and $41.2 \%$, respectively; both $P=0.001$ ). The chemical worker group also had a slightly higher rate of alcohol consumption. For example, $11.8 \%$ of the chemical workers reported consuming alcohol every day for the past 30 days, and $26.5 \%$ consumed alcohol 2-3 times per week; these percentages were higher than those of the organic worker group. Obesity and hypertension are potential metabolic risk factors that can influence thyroid hormone levels. However, there was no significant difference in the mean body mass index or blood pressure between the two groups. The TSH and FT3 concentrations were significantly different between the chemical workers and the organic workers (TSH: 1.58 vs $1.12 \mu \mathrm{IU} / \mathrm{mL}, P<0.001$ and FT3: 0.34 vs $0.31 \mathrm{ng} / \mathrm{dL}, P=0.001$, respectively); the difference in FT4 was not significant. The serum concentrations of TSH, T4, T3, FT4, and FT3 are routinely used to establish the presence of a thyroid disorder. In the current study, TSH, FT4, and FT3 were used to establish the prevalence of subclinical thyroid disease (either hyperthyroidism or hypothyroidism). Overall, 3.7\% of the chemical workers and $8.3 \%$ of the organic workers had subclinical thyroid disease. In addition, a high prevalence of other thyroid diseases was found among the chemical and organic worker groups (38.4\% and $42.4 \%$, respectively); however, the differences in prevalence between the groups was not significant $(P=0.055)$. Percent distribution of worker who wear partial personal protective equipment during pesticides application were reported with more than $50 \%$ wearing long shirts/ trousers $(92.1 \%)$ and rubber boots $(56.9 \%)$.

\section{Genetic Polymorphisms of Xenobiotic-Metabolizing Enzymes and Transporters Related to Pesticide Metabolism}

Pesticide biotransformation involves phase I and phase II enzymes, which carry out oxidative and conjugative reactions, respectively; also involved are phase III transporters, which carry out efflux mechanisms. The various types of pesticides used by the workers indicated the involvement of cytochrome P450, which has considerable catalytic diversity, along with other enzymes, including POR1, NAT1, NAT2, PON1, GSTP1, GSTM1, GSTT1, and ABCB1. The minor allele frequencies (MAFs) of these genes in the two groups are reported in Tables 2 and 3. No significant departures from HWE (at $P<0.05$ ) were observed for the 26 variants. The MAFs were similar among the two worker groups for CYP1A1 (rs1048943 and rs4646903), CYP1A2 (rs2069514 and rs762551), CYP3A5 rs776764, CYP2B6 rs2279343, CYP2D6 (rs1065852 and rs16947), CYP2E1 (rs3813867 and rs2031920), and POR1 (rs1057868 and rs1135612). However, some variant allele frequencies showed slight differences between the chemical and organic worker groups, such as CYP2B6 rs3745274 (0.27 and 0.39), CYP2C19 rs4244285 (0.37 and 0.22), and CYP2E1 rs6413432 (0.16 and 0.31, respectively) (Table 2).

The allele frequencies of phase II metabolizing enzymes (NAT1, NAT2, GST, and PON1) and the 
Table I Demographic Data of the Two Worker Groups

\begin{tabular}{|c|c|c|c|}
\hline \multirow[t]{2}{*}{ Age (mean), years } & Chemical Workers $(n=2 \mid 6)$ & Organic Workers $(n=229)$ & $P$ value \\
\hline & $51.1 \pm 9.8$ & $54.6 \pm 9.3$ & 0.004 \\
\hline \multicolumn{4}{|l|}{ Sex $(\%)$} \\
\hline Men & 161 (74.5\%) & $118(51.5 \%)$ & 0.001 \\
\hline Women & $55(25.5 \%)$ & 111 (48.5\%) & \\
\hline $\mathrm{BMI}, \mathrm{kg} / \mathrm{m}^{2}$ & $24.5 \pm 4.2$ & $23.8 \pm 6.2$ & 0.197 \\
\hline $\mathrm{SBP}, \mathrm{mmHg}$ & $132.1 \pm 16.9$ & $|30.6 \pm 1| .7$ & 0.897 \\
\hline $\mathrm{DBP}, \mathrm{mmHg}$ & $70.5 \pm 16.2$ & $71.7 \pm 17.9$ & 0.458 \\
\hline \multicolumn{4}{|l|}{ Smoking status, \% } \\
\hline Non-smokers & $145(68.4 \%)$ & $162(72.6 \%)$ & 0.001 \\
\hline Former smokers & $10(4.7 \%)$ & $25(11.2 \%)$ & \\
\hline Current smokers & $57(26.9 \%)$ & $36(16.1 \%)$ & \\
\hline Number of cigarettes/day & $11.32 \pm 6.3$ & $10.65 \pm 6.63$ & 0.627 \\
\hline Smoking duration, years & $25.89 \pm 13.2$ & $25.4 \pm 17.0$ & 0.886 \\
\hline \multicolumn{4}{|l|}{ Alcohol consumption, \% } \\
\hline No consumption & $70(32.9 \%)$ & $86(38.9 \%)$ & 0.001 \\
\hline Previously consumed & 7 (3.3\%) & 44 (19.9\%) & \\
\hline Currently consume & $136(63.8 \%)$ & 91 (4I.2\%) & \\
\hline Duration of alcohol consumption (years) & $21.13 \pm 12.27$ & $21.97 \pm 13.80$ & 0.650 \\
\hline \multicolumn{4}{|l|}{ Frequency of alcohol consumption } \\
\hline Everyday & $16(11.8 \%)$ & - & 0.001 \\
\hline 2-3 times/week & $36(26.5 \%)$ & $6(6.59 \%)$ & \\
\hline I time/month & $3(2.2 \%)$ & I (I.09\%) & \\
\hline Occasional & $8 \mathrm{I}(59.5 \%)$ & $84(92.31 \%)$ & \\
\hline $\mathrm{TSH}(\mu \mathrm{lU} / \mathrm{mL})^{\mathrm{a}}$ & $1.58(0.056-10.13)$ & $1.12(0.019-8.51)$ & $<0.001$ \\
\hline FT4 (ng/dL) ${ }^{a}$ & $0.83(0.55-1.63)$ & $0.82(0.52-1.21)$ & 0.531 \\
\hline $\mathrm{FT} 3(\mathrm{ng} / \mathrm{dL})^{\mathrm{a}}$ & $0.34(0.25-1.47)$ & $0.31(0.16-0.60)$ & 0.001 \\
\hline \multicolumn{4}{|l|}{ Prevalence of thyroid disorder (\%) } \\
\hline Normal thyroid function & $57.9 \%(n=125)$ & $49.3 \%(n=113)$ & 0.055 \\
\hline Subclinical thyroid disease & $3.7 \%(n=8)$ & $8.3 \%(n=19)$ & \\
\hline Other thyroid disorder & $38.4 \%(n=83)$ & $42.4 \%(n=97)$ & \\
\hline Personal protective equipment use (\%) & $93.9 \%(203)$ & - & - \\
\hline Wear rubber gloves & $28.2 \%(n=6 I)$ & & \\
\hline Wear goggle & $4.6 \%(n=10)$ & & \\
\hline Wear long shirts/ Trousers & $92.1 \%(=199)$ & & \\
\hline Wear rubber boots & $56.9 \%(n=123)$ & & \\
\hline Wear nose mask & $49.3 \%(n=95)$ & & \\
\hline Wear caps/hat & $40.7 \%(n=88)$ & & \\
\hline
\end{tabular}

Notes: ${ }^{a}$ Geometric mean and range. $P$ values by Student's $t$-test and Chi-square test.

Abbreviations: BMI, body mass index; DBP, diastolic blood pressure; FT3, free triiodothyronine; FT4, free thyroxine; SBP, systolic blood pressure; TSH, thyroid-stimulating hormone. 
Table 2 Allele Frequencies of CYP and POR Polymorphisms Among Chemical and Organic Workers

\begin{tabular}{|c|c|c|c|c|c|c|c|}
\hline \multirow[t]{2}{*}{ dbSNP ID } & \multirow[t]{2}{*}{ Gene } & \multirow[t]{2}{*}{ Allele } & \multirow[t]{2}{*}{ Function } & \multicolumn{4}{|c|}{ Minor Allele Frequency (\%) } \\
\hline & & & & Chemical Workers & HW P value & Organic Workers & HW $P$ value \\
\hline rsl048943 & CYPIAI & $\mathrm{A} / \mathrm{G}$ & Missense & 0.21 & 0.227 & 0.31 & 0.926 \\
\hline rs4646903 & CYPIAI & $\mathrm{T} / \mathrm{C}$ & Transcript variant & 0.49 & $0.78 \mathrm{I}$ & 0.42 & 0.109 \\
\hline rs20695I4 & CYPIA2 & $\mathrm{G} / \mathrm{A}$ & Transcript variant & 0.22 & 0.460 & 0.32 & 0.244 \\
\hline rs76255I & CYPIA2 & $\mathrm{C} / \mathrm{A}$ & Intron variant & 0.24 & 0.142 & 0.20 & 0.583 \\
\hline rs776746 & CYР3А5 & $\mathrm{A} / \mathrm{G}$ & Transcript variant & 0.42 & 0.071 & 0.40 & 0.156 \\
\hline rs3745274 & CYP2B6 & $\mathrm{G} / \mathrm{T}$ & Missense & 0.27 & 0.583 & 0.39 & 0.408 \\
\hline rs2279343 & CYP2B6 & $\mathrm{A} / \mathrm{G}$ & Missense & 0.22 & 0.128 & 0.27 & 0.176 \\
\hline rs4244285 & CYP2CI9 & $\mathrm{G} / \mathrm{A}$ & Synonymous variant & 0.37 & 0.638 & 0.22 & 0.761 \\
\hline rs1065852 & CYP2D6 & $\mathrm{C} / \mathrm{T}$ & Missense & 0.44 & 0.830 & 0.37 & 0.296 \\
\hline rs16947 & CYP2D6 & $\mathrm{G} / \mathrm{A}$ & Missense & 0.15 & 0.982 & 0.14 & 0.101 \\
\hline rs3813867 & CYP2EI & $\mathrm{G} / \mathrm{C}$ & Transcript variant & 0.19 & 0.783 & 0.12 & 0.164 \\
\hline rs2031920 & CYP2EI & $\mathrm{G} / \mathrm{C}$ & Transcript variant & 0.18 & 0.430 & 0.12 & 0.164 \\
\hline rs64I3432 & CYP2EI & T/A & Intron variant & 0.16 & 0.075 & 0.31 & 0.050 \\
\hline rs1057868 & PORI & $\mathrm{C} / \mathrm{T}$ & Missense & 0.31 & 0.820 & 0.32 & 0.329 \\
\hline rsl|356|2 & PORI & $A / G$ & Synonymous variant & 0.38 & 0.314 & 0.44 & 0.282 \\
\hline
\end{tabular}

Note: If $P<0.05$ in Chi-square test, it is not consistent with HWE.

Abbreviations: dbSNP, single nucleotide polymorphism database; HWE, Hardy-Weinberg equilibrium.

transporter $A B C B 1$ among the chemical and organic workers are shown in Table 3. The genetic polymorphisms of these genes generally showed the same distributions in the two groups, except for NAT1 rs1057126 (0.47 and 0.67), PON1 rs662 (0.38 and 0.23), and PON1 rs705379 (0.55 and 0.40 for the chemical and organic groups, respectively). Genotyping of the detoxifying enzyme GSTM1 found deletion in $64 \%$ of the chemical workers and $66 \%$ of organic workers. For
GSTT1, the null genotype was found in $33 \%$ and $25 \%$ of the chemical and organic workers, respectively.

\section{Thyroid Hormone Concentrations (TSH, FT4, and FT3) in Workers with Genetic Variations of Xenobiotic-Metabolizing Enzymes and Transporters}

Pesticides can act as thyroid-disrupting chemicals, and their metabolism in the body is partly determined by

Table 3 Allele Frequencies of NATI, NAT2, GST, PONI, and ABCBI Polymorphisms Among Chemical and Organic Workers

\begin{tabular}{|c|c|c|c|c|c|c|c|}
\hline \multirow[t]{2}{*}{ dBSNP ID } & \multirow[t]{2}{*}{ Gene } & \multirow[t]{2}{*}{ Allele } & \multirow[t]{2}{*}{ Function } & \multicolumn{4}{|c|}{ Minor Allele Frequency (\%) } \\
\hline & & & & Chemical Workers & HW P value & Organic Workers & HW P value \\
\hline rs|556| & NATI & $\mathrm{C} / \mathrm{A}$ & UTR variant & 0.45 & 0.052 & 0.48 & 0.656 \\
\hline rs $1057 \mid 26$ & NATI & T/A & UTR variant & 0.49 & 0.639 & 0.67 & 0.943 \\
\hline rs1041983 & NAT2 & $\mathrm{C} / \mathrm{T}$ & Synonymous variant & 0.55 & 0.209 & 0.50 & 0.098 \\
\hline rsI79993। & NAT2 & $\mathrm{G} / \mathrm{A}$ & Missense & 0.21 & 0.623 & 0.18 & 0.626 \\
\hline rs 1799930 & NAT2 & $\mathrm{G} / \mathrm{A}$ & Missense & 0.31 & 0.774 & 0.31 & 0.55 \\
\hline rs662 & PONI & $\mathrm{A} / \mathrm{G}$ & Missense & 0.38 & 0.92 & 0.23 & 0.053 \\
\hline rs705379 & PONI & $\mathrm{C} / \mathrm{T}$ & Transcript variant & 0.55 & 0.759 & 0.40 & 0.068 \\
\hline rs854560 & PONI & $\mathrm{G} / \mathrm{A}$ & Missense & 0.05 & 0.523 & 0.03 & 0.079 \\
\hline rs1045642 & $A B C B I$ & $\mathrm{C} / \mathrm{T}$ & Missense & 0.43 & 0.828 & 0.43 & 0.313 \\
\hline rsII28503 & $A B C B I$ & $\mathrm{C} / \mathrm{T}$ & Synonymous variant & 0.40 & 0.099 & 0.36 & 0.605 \\
\hline rsl695 & GSTPI & $\mathrm{A} / \mathrm{G}$ & Missense & 0.23 & 0.393 & 0.27 & 0.897 \\
\hline- & GSTMI & Null & Deletion & 0.64 & - & 0.66 & - \\
\hline- & GSTTI & Null & Deletion & 0.33 & - & 0.25 & - \\
\hline
\end{tabular}

Note: If $P<0.05$ in Chi-square test, it is not consistent with HWE.

Abbreviations: dbSNP, single nucleotide polymorphism database; HWE, Hardy-Weinberg equilibrium; UTR, untranslated region. 
genetic alterations in phase I and II enzymes and phase III transporters. We further analyzed the association between these polymorphisms and thyroid hormone concentrations (TSH, FT4, and FT3) (Tables 4-6). In the chemical worker group, individuals with a CYP1A1 rs1048943 heterozygous/variant genotype showed significantly lower TSH concentrations $(1.32 \pm 1.05 \mu \mathrm{IU} / \mathrm{mL})$ than those with the wild-type genotype $(1.69 \pm 0.98 \mu \mathrm{IU} /$ $\mathrm{mL}$ ); however, the TSH concentrations were higher than in individuals of the organic worker group who had the rs 1048943 genotype $(1.09 \pm 0.60 \mu \mathrm{IU} / \mathrm{mL}$, all $P<0.05)$. For those with CYP3A5 rs 776746, the TSH concentration was significantly different between the chemical and organic worker groups $(P<0.05)$. In this study, two SNPs of CYP2B6 (rs2279343 and rs3745274) were investigated; however, in chemical workers, only rs2279343 showed a significant difference in TSH concentration between those with the variant allele and those with the wild-type allele $(P<0.05)$. In addition, individuals in the organic worker group with heterozygous/ variant genotypes showed significantly lower TSH concentrations than those in the chemical worker group $(P<0.05) . C Y P 2 C 19$ is an enzyme involved in pesticide detoxification, and alterations in it may facilitate pathological disease as a consequence of occupational pesticide exposure. For CYP2C19 rs4244285, there was a significant difference in TSH concentration between chemical workers with the wild-type genotype and those with the heterozygous/variant genotype $(P<0.05)$. For those with the CYP2C19 rs4244285 heterozygous/ variant genotype, there was a significant difference in TSH concentration between the two worker groups $(P<0.05)$. For those with the CYP2D6 rs1065852 variation, there was also a significant difference in TSH concentration between chemical and organic worker groups $(1.65 \pm 0.74$ vs $1.25 \pm 0.61 \mu \mathrm{IU} / \mathrm{mL}, P<0.05)$; however,

Table 4 Thyroid Hormone Concentrations Among Chemical and Organic Workers with CYP Polymorphisms

\begin{tabular}{|c|c|c|c|c|c|c|c|}
\hline \multicolumn{2}{|l|}{ Gene } & \multicolumn{2}{|c|}{ TSH $(\mu \mathrm{IU} / \mathrm{mL})$} & \multicolumn{2}{|c|}{ FT4 (ng/dL) } & \multicolumn{2}{|c|}{ FT3 (ng/dL) } \\
\hline Gene & Allele & $\begin{array}{l}\text { Chemical } \\
\text { Workers }\end{array}$ & $\begin{array}{l}\text { Organic } \\
\text { Workers }\end{array}$ & $\begin{array}{l}\text { Chemical } \\
\text { Workers }\end{array}$ & $\begin{array}{l}\text { Organic } \\
\text { Workers }\end{array}$ & $\begin{array}{l}\text { Chemical } \\
\text { Workers }\end{array}$ & $\begin{array}{l}\text { Organic } \\
\text { Workers }\end{array}$ \\
\hline $\begin{array}{l}\text { CYPIAI } \\
\text { rsI048943 }\end{array}$ & $\begin{array}{c}\mathrm{AA} \\
\mathrm{AG} / \mathrm{GG}\end{array}$ & $\begin{array}{c}1.69 \pm 0.98 \\
1.32 \pm 1.05 \#\end{array}$ & $\begin{array}{l}1.15 \pm 1.10 \\
1.09 \pm 0.60 *\end{array}$ & $\begin{array}{c}0.84 \pm 0.13 \\
0.69 \pm 0.16 \#\end{array}$ & $\begin{array}{l}0.81 \pm 0.11 \\
0.83 \pm 0.13\end{array}$ & $\begin{array}{l}0.34 \pm 0.04 \\
0.34 \pm 0.12\end{array}$ & $\begin{array}{l}0.3 \mid \pm 0.07 \\
0.3 \mid \pm 0.06\end{array}$ \\
\hline $\begin{array}{l}\text { CYPIAI } \\
\text { rs4646903 }\end{array}$ & $\begin{array}{c}\mathrm{TT} \\
\mathrm{TC} / \mathrm{CC}\end{array}$ & $\begin{array}{l}1.64 \pm 1.05 \\
1.56 \pm 1.19\end{array}$ & $\begin{array}{l}1.32 \pm 1.02 \\
1.26 \pm 0.77\end{array}$ & $\begin{array}{l}0.82 \pm 0.14 \\
0.84 \pm 0.15\end{array}$ & $\begin{array}{l}0.83 \pm 0.44 \\
0.82 \pm 0.12\end{array}$ & $\begin{array}{l}0.35 \pm 0.15 \\
0.33 \pm 0.04\end{array}$ & $\begin{array}{l}0.32 \pm 0.05 \\
0.3 I \pm 0.07\end{array}$ \\
\hline $\begin{array}{l}\text { CYPIA2 } \\
\text { rs20695I4 }\end{array}$ & $\begin{array}{c}\text { GG } \\
\text { GA/AA }\end{array}$ & $\begin{array}{l}1.52 \pm 1.00 \\
1.65 \pm 1.35\end{array}$ & $\begin{array}{l}1.36 \pm 0.81 \\
1.24 \pm 0.92\end{array}$ & $\begin{array}{l}0.84 \pm 0.13 \\
0.82 \pm 0.16\end{array}$ & $\begin{array}{l}0.82 \pm 0.12 \\
0.82 \pm 0.13\end{array}$ & $\begin{array}{l}0.34 \pm 0.04 \\
0.34 \pm 0.12\end{array}$ & $\begin{array}{l}0.3 I \pm 0.07 \\
0.3 I \pm 0.06\end{array}$ \\
\hline $\begin{array}{l}\text { CYPIA2 } \\
\text { rs76255I }\end{array}$ & $\begin{array}{c}C C \\
C A / A A\end{array}$ & $\begin{array}{l}1.65 \pm 1.10 \\
1.48 \pm 1.23\end{array}$ & $\begin{array}{l}1.42 \pm 0.72 \\
1.21 \pm 1.10\end{array}$ & $\begin{array}{l}0.8 I \pm 0.15 \\
0.86 \pm 0.14\end{array}$ & $\begin{array}{c}0.83 \pm 0.13 \\
0.8 I \pm 0.12 *\end{array}$ & $\begin{array}{l}0.38 \pm 0.04 \\
0.34 \pm 0.12\end{array}$ & $\begin{array}{l}0.31 \pm 0.06 \\
0.32 \pm 0.06\end{array}$ \\
\hline $\begin{array}{l}\text { CYP3A5 } \\
\text { rs776746 }\end{array}$ & $\begin{array}{c}\text { AA } \\
A G / G G\end{array}$ & $\begin{array}{l}I .62 \pm I .4 \mid \\
I .60 \pm 0.96\end{array}$ & $\begin{array}{l}1.23 \pm 0.73 \\
1.18 \pm 0.98 *\end{array}$ & $\begin{array}{l}0.85 \pm 0.17 \\
0.82 \pm 0.12\end{array}$ & $\begin{array}{l}0.84 \pm 0.14 \\
0.82 \pm 0.12\end{array}$ & $\begin{array}{l}0.35 \pm 0.13 \\
0.33 \pm 0.03\end{array}$ & $\begin{array}{l}0.32 \pm 0.07 \\
0.31 \pm 0.06\end{array}$ \\
\hline $\begin{array}{l}\text { CYP2B6 } \\
\text { rs2279343 }\end{array}$ & $\begin{array}{c}\mathrm{AA} \\
\mathrm{AG} / \mathrm{GG}\end{array}$ & $\begin{array}{c}|.62 \pm| .3 \mid \\
|.48 \pm| .2 \mid \#\end{array}$ & $\begin{array}{l}1.04 \pm 0.79 \\
1.21 \pm 1.12 *\end{array}$ & $\begin{array}{l}0.83 \pm 0.12 \\
0.83 \pm 0.17\end{array}$ & $\begin{array}{l}0.83 \pm 0.13 \\
0.82 \pm 0.12\end{array}$ & $\begin{array}{l}0.34 \pm 0.04 \\
0.35 \pm 0.11\end{array}$ & $\begin{array}{c}0.37 \pm 0.07 \\
0.31 \pm 0.09 *\end{array}$ \\
\hline $\begin{array}{l}\text { CYP2B6 } \\
\text { rs3745274 }\end{array}$ & $\begin{array}{c}\text { GG } \\
\text { GT/TT }\end{array}$ & $\begin{array}{l}1.64 \pm 0.94 \\
1.49 \pm 1.03\end{array}$ & $\begin{array}{l}1.14 \pm 0.79 \\
1.28 \pm 0.92\end{array}$ & $\begin{array}{l}0.83 \pm 0.15 \\
0.83 \pm 0.14\end{array}$ & $\begin{array}{l}0.82 \pm 0.12 \\
0.82 \pm 0.13\end{array}$ & $\begin{array}{l}0.34 \pm 0.11 \\
0.33 \pm 0.03\end{array}$ & $\begin{array}{l}0.3 I \pm 0.06 \\
0.3 I \pm 0.06\end{array}$ \\
\hline $\begin{array}{l}\text { CYP2C19 } \\
\text { rs4244285 }\end{array}$ & $\begin{array}{c}\text { GG } \\
\text { GA/AA }\end{array}$ & $\begin{array}{c}1.87 \pm 0.90 \\
1.32 \pm 0.84 \#\end{array}$ & $\begin{array}{l}1.43 \pm 0.72 \\
1.25 \pm 1.05 *\end{array}$ & $\begin{array}{c}0.80 \pm 0.16 \\
0.65 \pm 0.18 \#\end{array}$ & $\begin{array}{l}0.81 \pm 0.18 \\
0.78 \pm 0.21\end{array}$ & $\begin{array}{l}0.34 \pm 0.12 \\
0.35 \pm 0.09\end{array}$ & $\begin{array}{c}0.32 \pm 0.09 \\
0.28 \pm 0.06 *\end{array}$ \\
\hline $\begin{array}{l}\text { CYP2D6 } \\
\text { rs1065852 }\end{array}$ & $\begin{array}{c}\mathrm{CC} \\
\mathrm{CT} / \mathrm{TT}\end{array}$ & $\begin{array}{c}1.42 \pm 0.86 \\
1.65 \pm 0.74\end{array}$ & $\begin{array}{c}1.27 \pm 0.83 \\
1.25 \pm 0.6 I^{*}\end{array}$ & $\begin{array}{l}0.84 \pm 0.16 \\
0.82 \pm 0.13\end{array}$ & $\begin{array}{l}0.83 \pm 0.12 \\
0.82 \pm 0.13\end{array}$ & $\begin{array}{l}0.34 \pm 0.05 \\
0.33 \pm 0.04\end{array}$ & $\begin{array}{l}0.31 \pm 0.04 \\
0.32 \pm 0.06\end{array}$ \\
\hline $\begin{array}{l}\text { CYP2D6 } \\
\text { rsI6947 }\end{array}$ & $\begin{array}{c}\text { GG } \\
\text { GA/AA }\end{array}$ & $\begin{array}{l}1.60 \pm 1.26 \\
1.52 \pm 0.83\end{array}$ & $\begin{array}{l}|.49 \pm 0.7| \\
\mid .30 \pm 0.72\end{array}$ & $\begin{array}{l}0.84 \pm 0.15 \\
0.82 \pm 0.12\end{array}$ & $\begin{array}{l}0.83 \pm 0.12 \\
0.8 I \pm 0.13\end{array}$ & $\begin{array}{l}0.34 \pm 0.10 \\
0.33 \pm 0.03\end{array}$ & $\begin{array}{l}0.31 \pm 0.06 \\
0.32 \pm 0.06\end{array}$ \\
\hline
\end{tabular}

Notes: "Significant difference from wild-type genotype by Student's $t$-test; at $P<0.05$. *Significant difference from chemical worker group by Student's $t$-test at $P<0.05$. Abbreviations: FT3, free triiodothyronine; FT4, free thyroxine; TSH, thyroid-stimulating hormone. 
Table 5 Thyroid Hormone Concentrations Among Chemical and Organic Workers with CYP2EI, PORI, NATI, and NAT2 Polymorphisms

\begin{tabular}{|c|c|c|c|c|c|c|c|}
\hline \multicolumn{2}{|l|}{ Gene } & \multicolumn{2}{|c|}{ TSH $(\mu \mathrm{IU} / \mathrm{mL})$} & \multicolumn{2}{|c|}{ FT4 (ng/dL) } & \multicolumn{2}{|c|}{ FT3 (ng/dL) } \\
\hline Gene & Allele & $\begin{array}{l}\text { Chemical } \\
\text { Workers }\end{array}$ & $\begin{array}{l}\text { Organic } \\
\text { Workers }\end{array}$ & $\begin{array}{l}\text { Chemical } \\
\text { Workers }\end{array}$ & $\begin{array}{l}\text { Organic } \\
\text { Workers }\end{array}$ & $\begin{array}{l}\text { Chemical } \\
\text { Workers }\end{array}$ & $\begin{array}{c}\text { Organic } \\
\text { Workers }\end{array}$ \\
\hline $\begin{array}{l}\text { CYP2EI } \\
\text { rs38I } 3867\end{array}$ & $\begin{array}{c}\mathrm{GG} \\
\mathrm{GC} / \mathrm{CC}\end{array}$ & $\begin{array}{l}1.62 \pm 1.10 \\
1.50 \pm 1.25\end{array}$ & $\begin{array}{l}1.42 \pm 0.95 \\
1.23 \pm 0.97\end{array}$ & $\begin{array}{l}0.85 \pm 0.16 \\
0.87 \pm 0.11\end{array}$ & $\begin{array}{l}0.83 \pm 0.12 \\
0.81 \pm 0.14\end{array}$ & $\begin{array}{l}0.34 \pm 0.10 \\
0.34 \pm 0.04\end{array}$ & $\begin{array}{l}0.31 \pm 0.06 \\
0.32 \pm 0.07\end{array}$ \\
\hline $\begin{array}{l}\text { CYP2EI } \\
\text { rs2031920 }\end{array}$ & $\begin{array}{c}\mathrm{GG} \\
\mathrm{GC} / \mathrm{CC}\end{array}$ & $\begin{array}{l}1.53 \pm 1.10 \\
1.48 \pm 1.25\end{array}$ & $\begin{array}{l}1.32 \pm 0.75 \\
1.35 \pm 0.74\end{array}$ & $\begin{array}{l}0.84 \pm 0.16 \\
0.81 \pm 0.19\end{array}$ & $\begin{array}{l}0.82 \pm 0.12 \\
0.82 \pm 0.14\end{array}$ & $\begin{array}{l}0.34 \pm 0.10 \\
0.34 \pm 0.07\end{array}$ & $\begin{array}{l}0.32 \pm 0.06 \\
0.33 \pm 0.08\end{array}$ \\
\hline $\begin{array}{l}\text { CYP2EI } \\
\text { rs64I3432 }\end{array}$ & $\begin{array}{c}\text { TT } \\
\text { TA/AA }\end{array}$ & $\begin{array}{c}1.52 \pm 1.26 \\
1.29 \pm 0.87^{\#}\end{array}$ & $\begin{array}{l}1.24 \pm 0.59 \\
1.38 \pm 1.04\end{array}$ & $\begin{array}{l}0.82 \pm 0.15 \\
0.86 \pm 0.13\end{array}$ & $\begin{array}{c}0.83 \pm 0.13 \\
0.8 I \pm 0.12 *\end{array}$ & $\begin{array}{l}0.33 \pm 0.04 \\
0.36 \pm 0.14\end{array}$ & $\begin{array}{l}0.32 \pm 0.07 \\
0.32 \pm 0.06\end{array}$ \\
\hline $\begin{array}{l}\text { PORI } \\
\text { rsI057868 }\end{array}$ & $\begin{array}{c}\mathrm{CC} \\
\mathrm{CT} / \mathrm{TT}\end{array}$ & $\begin{array}{l}1.64 \pm 1.19 \\
1.50 \pm 1.11\end{array}$ & $\begin{array}{l}1.39 \pm 0.56 \\
1.32 \pm 1.05\end{array}$ & $\begin{array}{l}0.83 \pm 0.14 \\
0.83 \pm 0.15\end{array}$ & $\begin{array}{l}0.81 \pm 0.12 \\
0.83 \pm 0.13\end{array}$ & $\begin{array}{l}0.35 \pm 0.12 \\
0.33 \pm 0.03\end{array}$ & $\begin{array}{l}0.32 \pm 0.06 \\
0.32 \pm 0.13\end{array}$ \\
\hline $\begin{array}{l}\text { PORI } \\
\text { rsII356I2 }\end{array}$ & $\begin{array}{c}A A \\
A G / G G\end{array}$ & $\begin{array}{l}1.56 \pm 1.32 \\
1.52 \pm 1.03\end{array}$ & $\begin{array}{l}1.38 \pm 0.45 \\
1.24 \pm 0.57\end{array}$ & $\begin{array}{l}0.83 \pm 0.15 \\
0.83 \pm 0.14\end{array}$ & $\begin{array}{l}0.82 \pm 0.14 \\
0.82 \pm 0.12\end{array}$ & $\begin{array}{l}0.34 \pm 0.12 \\
0.33 \pm 0.04\end{array}$ & $\begin{array}{l}0.32 \pm 0.06 \\
0.31 \pm 0.06\end{array}$ \\
\hline $\begin{array}{l}\text { NATI } \\
\text { rsI556I }\end{array}$ & $\begin{array}{c}\mathrm{CC} \\
\mathrm{CA} / \mathrm{AA}\end{array}$ & $\begin{array}{l}1.52 \pm 1.09 \\
1.56 \pm 1.02\end{array}$ & $\begin{array}{l}I .38 \pm 1.01 \\
I .14 \pm 0.4 I^{*}\end{array}$ & $\begin{array}{l}0.79 \pm 0.12 \\
0.85 \pm 0.15\end{array}$ & $\begin{array}{c}0.80 \pm 0.12 \\
0.8 I \pm 0.12 *\end{array}$ & $\begin{array}{l}0.33 \pm 0.03 \\
0.34 \pm 0.10\end{array}$ & $\begin{array}{c}0.29 \pm 0.07 \\
0.32 \pm 0.06 *\end{array}$ \\
\hline $\begin{array}{l}\text { NATI } \\
\text { rsI057I26 }\end{array}$ & $\begin{array}{c}\text { TT } \\
\text { TA/AA }\end{array}$ & $\begin{array}{l}1.56 \pm 0.83 \\
1.65 \pm 1.12\end{array}$ & $\begin{array}{l}\text { I. } 28 \pm 0.92 \\
\text { I. } 12 \pm 0.90 *\end{array}$ & $\begin{array}{c}0.90 \pm 0.17 \\
0.80 \pm 0.13^{\#}\end{array}$ & $\begin{array}{l}0.85 \pm 0.12 \\
0.82 \pm 0.13\end{array}$ & $\begin{array}{l}0.34 \pm 0.04 \\
0.34 \pm 0.09\end{array}$ & $\begin{array}{l}0.32 \pm 0.05 \\
0.31 \pm 0.06\end{array}$ \\
\hline $\begin{array}{l}\text { NAT2 } \\
\text { rsI04I983 }\end{array}$ & $\begin{array}{c}\mathrm{CC} \\
\mathrm{CT} / \mathrm{TT}\end{array}$ & $\begin{array}{c}1.27 \pm 0.78 \\
1.65 \pm 0.57^{\#}\end{array}$ & $\begin{array}{l}1.36 \pm 1.24 \\
|.3| \pm 0.74\end{array}$ & $\begin{array}{l}0.82 \pm 0.14 \\
0.83 \pm 0.15\end{array}$ & $\begin{array}{l}0.84 \pm 0.15 \\
0.82 \pm 0.12\end{array}$ & $\begin{array}{l}0.33 \pm 0.04 \\
0.34 \pm 0.09\end{array}$ & $\begin{array}{l}0.32 \pm 0.07 \\
0.31 \pm 0.06\end{array}$ \\
\hline $\begin{array}{l}\text { NAT2 } \\
\text { rsI79993I }\end{array}$ & $\begin{array}{c}\text { GG } \\
\text { GA/AA }\end{array}$ & $\begin{array}{c}1.57 \pm 1.09 \\
2.13 \pm 1.32^{\#}\end{array}$ & $\begin{array}{l}1.17 \pm 0.96 \\
1.02 \pm 0.87\end{array}$ & $\begin{array}{c}0.82 \pm 0.14 \\
0.51 \pm 0.15^{\#}\end{array}$ & $\begin{array}{l}0.83 \pm 0.14 \\
0.80 \pm 0.10\end{array}$ & $\begin{array}{l}0.34 \pm 0.10 \\
0.33 \pm 0.03\end{array}$ & $\begin{array}{l}0.32 \pm 0.07 \\
0.30 \pm 0.12\end{array}$ \\
\hline $\begin{array}{l}\text { NAT2 } \\
\text { rsI799930 }\end{array}$ & $\begin{array}{c}\text { GG } \\
\text { GA/AA }\end{array}$ & $\begin{array}{c}\mathrm{I} .45 \pm 0.74 \\
\mathrm{I} .70 \pm 0.63^{\#}\end{array}$ & $\begin{array}{l}1.29 \pm 0.43 \\
1.14 \pm 0.96\end{array}$ & $\begin{array}{l}0.84 \pm 0.15 \\
0.82 \pm 0.14\end{array}$ & $\begin{array}{l}0.82 \pm 0.12 \\
0.82 \pm 0.13\end{array}$ & $\begin{array}{l}0.35 \pm 0.14 \\
0.34 \pm 0.11\end{array}$ & $\begin{array}{l}0.30 \pm 0.10 \\
0.33 \pm 0.06\end{array}$ \\
\hline
\end{tabular}

Notes: ${ }^{\#}$ Significant difference from wild-type genotype by Student's $t$-test; at $\mathrm{P}<0.05$. *Significant difference from chemical worker group by Student's $t$-test at $P<0.05$. Abbreviations: FT3, free triiodothyronine; FT4, free thyroxine; TSH, thyroid-stimulating hormone.

there was not a significant difference in TSH concentration between the groups for those with the CYP2D6 rs16947 genotype.

For those with the heterozygous/variant genotypes of CYP1A2 rs762551, CYP2B6 rs2279343, and CYP2C19 rs4244285, the chemical workers had significantly higher FT4 and FT3 concentrations compared with the organic workers (all $P<0.05$ ). Furthermore, in the chemical worker group, those with the CYP2C19 rs4244285 variant had significantly lower FT4 concentrations than those with the wildtype genotype ( $0.65 \pm 0.18$ vs $0.80 \pm 0.16 \mathrm{ng} / \mathrm{dL}, P<0.05$ ).

Table 5 shows the thyroid hormone levels of workers with CYP2E1, POR1, NAT1, and NAT2 polymorphisms. Chemical workers with the CYP2E1 rs6413432 and NAT2 rs1041983, rs1799931, and rs1799930 heterozygous/variant genotypes exhibited significant differences in TSH concentrations compared with those with wild-type genotype (all $P<0.05$ ). Comparing the TSH concentrations in the two worker groups revealed the influence of the NAT1 rs15561 and rs1057126 heterozygous/variant genotypes $(P<0.05)$. In chemical workers, the FT4 concentrations were lower in those with NAT1 rs1057126 and NAT2 rs1799931 heterozygous/variant genotypes compared with those with the wild-type genotype. Regarding the FT3 concentration, similar findings were found for the CYP2E1 rs6413432 and NAT1 rs15561 genotypes (all $P<0.05$ ).

The thyroid hormone concentrations in workers with genetic polymorphisms of PON1, ABCB1, and GSTs are presented in Table 6 . The xenobiotic transporter $A B C B 1$, with two SNPs (rs1045642 and rs1128503), was investigated to assess its impact on the HPT axis in workers exposed to pesticides. The relationship between PON1 SNPs and thyroid hormone concentrations of occupationally exposed workers was investigated. Within the 
Table 6 Thyroid Hormone Concentrations Among Chemical and Organic Workers with PONI, ABCBI, and GST Polymorphisms

\begin{tabular}{|c|c|c|c|c|c|c|c|}
\hline \multicolumn{2}{|l|}{ Gene } & \multicolumn{2}{|c|}{ TSH $(\mu / \mathrm{U} / \mathrm{mL})$} & \multicolumn{2}{|c|}{ FT4 (ng/dL) } & \multicolumn{2}{|c|}{ FT3 (ng/dL) } \\
\hline Gene & Allele & $\begin{array}{l}\text { Chemical } \\
\text { Workers }\end{array}$ & $\begin{array}{l}\text { Organic } \\
\text { Workers }\end{array}$ & $\begin{array}{l}\text { Chemical } \\
\text { Workers }\end{array}$ & $\begin{array}{l}\text { Organic } \\
\text { Workers }\end{array}$ & $\begin{array}{l}\text { Chemical } \\
\text { Workers }\end{array}$ & $\begin{array}{l}\text { Organic } \\
\text { Workers }\end{array}$ \\
\hline $\begin{array}{l}\text { PONI } \\
\text { rs662 }\end{array}$ & $\begin{array}{c}A A \\
A G / G G\end{array}$ & $\begin{array}{c}1.65 \pm 1.16 \\
1.06 \pm 0.87^{\#}\end{array}$ & $\begin{array}{l}1.29 \pm 0.69 \\
1.02 \pm 0.65\end{array}$ & $\begin{array}{c}0.82 \pm 0.19 \\
0.67 \pm 0.34^{\#}\end{array}$ & $\begin{array}{l}0.8 I \pm 0.11 \\
0.82 \pm 0.14\end{array}$ & $\begin{array}{l}0.34 \pm 0.13 \\
0.37 \pm 0.18\end{array}$ & $\begin{array}{l}0.30 \pm 0.09 \\
0.31 \pm 0.12\end{array}$ \\
\hline $\begin{array}{l}\text { PONI } \\
\text { rs705379 }\end{array}$ & $\begin{array}{c}\mathrm{CC} \\
\mathrm{CT} / \mathrm{TT}\end{array}$ & $\begin{array}{c}1.81 \pm 0.68 \\
1.42 \pm 0.74^{\#}\end{array}$ & $\begin{array}{c}1.22 \pm 1.03 \\
1.06 \pm 0.76 *\end{array}$ & $\begin{array}{l}0.82 \pm 0.11 \\
0.83 \pm 0.15\end{array}$ & $\begin{array}{l}0.83 \pm 0.12 \\
0.82 \pm 0.13\end{array}$ & $\begin{array}{c}0.36 \pm 0.03 \\
0.30 \pm 0.09^{\#}\end{array}$ & $\begin{array}{l}0.32 \pm 0.06 \\
0.31 \pm 0.06\end{array}$ \\
\hline $\begin{array}{l}\text { PONI } \\
\text { rs854560 }\end{array}$ & $\begin{array}{c}\text { GG } \\
\text { GA/AA }\end{array}$ & $\begin{array}{l}1.62 \pm 0.87 \\
1.36 \pm 0.73^{\#}\end{array}$ & $\begin{array}{l}1.32 \pm 0.89 \\
1.28 \pm 0.44\end{array}$ & $\begin{array}{l}0.83 \pm 0.14 \\
0.86 \pm 0.22\end{array}$ & $\begin{array}{l}0.82 \pm 0.12 \\
0.89 \pm 0.15\end{array}$ & $\begin{array}{l}0.34 \pm 0.09 \\
0.34 \pm 0.03\end{array}$ & $\begin{array}{l}0.3 I \pm 0.06 \\
0.36 \pm 0.09\end{array}$ \\
\hline $\begin{array}{l}\text { PONI rs662/ } \\
\text { rs705379/ } \\
\text { rs854560 }\end{array}$ & $\begin{array}{c}\text { Normal } \\
\text { At least I } \\
\text { variant } \\
\geq 2 \text { variants }\end{array}$ & $\begin{array}{l}1.50 \pm 0.75 \\
1.49 \pm 0.94 \\
1.43 \pm 0.69\end{array}$ & $\begin{array}{l}1.32 \pm 1.03 \\
1.29 \pm 0.93 \\
1.38 \pm 0.64\end{array}$ & $\begin{array}{l}0.82 \pm 0.11 \\
0.8 I \pm 0.13 \\
0.84 \pm 0.16\end{array}$ & $\begin{array}{l}0.83 \pm 0.12 \\
0.79 \pm 0.14 \\
0.84 \pm 0.12\end{array}$ & $\begin{array}{l}0.33 \pm 0.03 \\
0.36 \pm 0.18 \\
0.34 \pm 0.04\end{array}$ & $\begin{array}{l}0.32 \pm 0.06 \\
0.3 I \pm 0.05 \\
0.3 I \pm 0.07\end{array}$ \\
\hline $\begin{array}{l}A B C B I \\
\text { rsI045642 }\end{array}$ & $\begin{array}{c}\mathrm{CC} \\
\mathrm{CT} / \mathrm{TT}\end{array}$ & $\begin{array}{l}1.56 \pm 0.88 \\
1.59 \pm 0.69\end{array}$ & $\begin{array}{l}1.59 \pm 1.26 \\
1.15 \pm 0.99 *\end{array}$ & $\begin{array}{l}0.85 \pm 0.17 \\
0.82 \pm 0.13\end{array}$ & $\begin{array}{l}0.83 \pm 0.13 \\
0.82 \pm 0.12\end{array}$ & $\begin{array}{l}0.33 \pm 0.03 \\
0.34 \pm 0.10\end{array}$ & $\begin{array}{l}0.31 \pm 0.05 \\
0.31 \pm 0.04\end{array}$ \\
\hline $\begin{array}{l}A B C B \mid \\
\text { rs I I 28503 }\end{array}$ & $\begin{array}{c}\text { CC } \\
\text { CT/TT }\end{array}$ & $\begin{array}{l}\text { I. } 34 \pm 0.42 \\
\text { I. } 64 \pm 0.67^{\#}\end{array}$ & $\begin{array}{l}1.25 \pm 1.19 \\
1.03 \pm 0.55^{*}\end{array}$ & $\begin{array}{c}0.84 \pm 0.15 \\
0.72 \pm 0.09^{\#}\end{array}$ & $\begin{array}{l}0.82 \pm 0.12 \\
0.82 \pm 0.13\end{array}$ & $\begin{array}{l}0.35 \pm 0.14 \\
0.35 \pm 0.03\end{array}$ & $\begin{array}{l}0.32 \pm 0.12 \\
0.31 \pm 0.06\end{array}$ \\
\hline $\begin{array}{l}\text { GSTPI } \\
\text { rsI695 }\end{array}$ & $\begin{array}{c}A A \\
A G / G G\end{array}$ & $\begin{array}{l}1.59 \pm 1.27 \\
1.56 \pm 0.97\end{array}$ & $\begin{array}{l}1.33 \pm 0.94 \\
I . I I \pm 0.8 I\end{array}$ & $\begin{array}{l}0.83 \pm 0.14 \\
0.84 \pm 0.15\end{array}$ & $\begin{array}{l}0.82 \pm 0.13 \\
0.82 \pm 0.12\end{array}$ & $\begin{array}{l}0.34 \pm 0.11 \\
0.33 \pm 0.05\end{array}$ & $\begin{array}{l}0.31 \pm 0.06 \\
0.31 \pm 0.06\end{array}$ \\
\hline GSTMI & $\begin{array}{l}\text { Present } \\
\text { Null }\end{array}$ & $\begin{array}{l}1.26 \pm 0.68 \\
1.52 \pm 1.1 I^{\#}\end{array}$ & $\begin{array}{l}1.35 \pm 1.03 \\
1.47 \pm 0.79\end{array}$ & $\begin{array}{c}0.85 \pm 0.15 \\
0.74 \pm 0.27^{\#}\end{array}$ & $\begin{array}{l}0.83 \pm 0.13 \\
0.82 \pm 0.12\end{array}$ & $\begin{array}{l}0.35 \pm 0.14 \\
0.38 \pm 0.17\end{array}$ & $\begin{array}{c}0.3 I \pm 0.1 \mathrm{I} \\
0.28 \pm 0.10^{*}\end{array}$ \\
\hline GSTTI & $\begin{array}{c}\text { Present } \\
\text { Null }\end{array}$ & $\begin{array}{c}1.49 \pm 0.9 \mid \\
1.75 \pm 0.78^{\#}\end{array}$ & $\begin{array}{l}\mathrm{I} .31 \pm 0.9 \mathrm{I} \\
\mathrm{I} .45 \pm 0.75\end{array}$ & $\begin{array}{l}0.89 \pm 0.15 \\
0.84 \pm 0.14\end{array}$ & $\begin{array}{l}0.82 \pm 0.12 \\
0.83 \pm 0.14\end{array}$ & $\begin{array}{l}0.34 \pm 0.10 \\
0.37 \pm 0.04\end{array}$ & $\begin{array}{l}0.31 \pm 0.06 \\
0.30 \pm 0.04\end{array}$ \\
\hline $\begin{array}{l}\text { GSTTI/ } \\
\text { GSTI } \\
\text { IGSTPI }\end{array}$ & $\begin{array}{l}\text { Normal } \\
\text { At least } \\
\text { I variant } \\
\geq 2 \text { variants }\end{array}$ & $\begin{array}{l}1.67 \pm 0.98 \\
1.51 \pm 1.05 \\
1.56 \pm 0.57\end{array}$ & $\begin{array}{l}1.48 \pm 1.03 \\
1.35 \pm 0.94 \\
1.39 \pm 0.9 \mid\end{array}$ & $\begin{array}{c}0.85 \pm 0.13 \\
0.8 I \pm 0.13 \\
0.84 \pm 0.16\end{array}$ & $\begin{array}{l}0.85 \pm 0.16 \\
0.81 \pm 0.12 \\
0.83 \pm 0.12\end{array}$ & $\begin{array}{l}0.33 \pm 0.03 \\
0.33 \pm 0.03 \\
0.34 \pm 0.12\end{array}$ & $\begin{array}{l}0.30 \pm 0.06 \\
0.31 \pm 0.06\end{array}$ \\
\hline
\end{tabular}

Notes: ${ }^{\#}$ Significant difference from wild-type genotype by Student's $t$-test and ANOVA; at $P<0.05$. *Significant difference from chemical worker group by Student's $t$-test at $P<0.05$.

Abbreviations: FT3, free triiodothyronine; FT4, free thyroxine; TSH, thyroid-stimulating hormone.

chemical worker group, individuals with the heterozygous/ variant genotypes PON1 rs662, rs705379, and rs854560 had lower TSH concentrations than those with the wildtype genotype, and the inverse association was noted for those with $A B C B 1$ rs1128503, GSTM1, and GSTT1 variations. Comparing the chemical and organic worker groups revealed significant differences (all $P<0.05$ ) in TSH concentrations related to the PON1 rs705379 and $A B C B 1$ (rs1045642 and rs1128503) genotypes. For workers with these heterozygous/variant genotypes, the FT4 concentrations were significantly lower in chemical workers than in organic workers $(P<0.05)$. Changes in the FT3 concentrations were affected by the PON1 rs705379 and GSTM1 genetic variations $(P<0.05)$.

\section{ORs and AORs of Thyroid Dysfunction Related to Genetic Variation of Xenobiotic-Metabolizing Enzymes and Transporters Among Chemical and Organic Workers}

Logistic regression analysis was used to calculate the ORs, AORs, and 95\% confidence intervals (CIs) for thyroid dysfunction in workers with heterozygous/variant genotypes of these enzymes and transporters, using the wild-type genotype as a reference (Table 7). The risk of developing subclinical thyroid disease or other thyroid disease was investigated for those with CYP enzyme polymorphisms. Only three CYP genes (CYP1A1 rs1048943, CYP2B6 
Table 7 OR and Adjusted or $(95 \% \mathrm{Cl})$ of Thyroid Dysfunction Among Chemical and Organic Workers with CPY, NAT2, and PONI Polymorphisms

\begin{tabular}{|c|c|c|c|c|c|c|c|}
\hline \multirow[t]{2}{*}{ Gene } & \multirow[t]{2}{*}{ Allele } & \multicolumn{3}{|c|}{ OR $(95 \% \mathrm{CI})$} & \multicolumn{3}{|c|}{ Adjusted OR* $(95 \% \mathrm{Cl})$} \\
\hline & & $\begin{array}{c}\text { All } \\
\text { Workers }\end{array}$ & $\begin{array}{l}\text { Chemical } \\
\text { Workers }\end{array}$ & $\begin{array}{l}\text { Organic } \\
\text { Workers }\end{array}$ & $\begin{array}{c}\text { All } \\
\text { Workers }\end{array}$ & $\begin{array}{l}\text { Chemical } \\
\text { Workers }\end{array}$ & $\begin{array}{l}\text { Organic } \\
\text { Workers }\end{array}$ \\
\hline $\begin{array}{l}\text { CYPIAI } \\
\text { rsI048943 }\end{array}$ & $\begin{array}{c}A A \\
A G / G G\end{array}$ & $\begin{array}{c}1.00 \\
1.32 \\
(0.73-2.38)\end{array}$ & $\begin{array}{c}1.00 \\
18.14- \\
(2.29-143.2)\end{array}$ & $\begin{array}{c}1.00 \\
0.58 \\
(0.28-1.18)\end{array}$ & $\begin{array}{c}1.00 \\
1.08 \\
(0.65-3.02)\end{array}$ & $\begin{array}{c}1.00 \\
15.12 \\
(2.01-134.6)\end{array}$ & $\begin{array}{c}1.00 \\
0.58 \\
(0.47-2.01)\end{array}$ \\
\hline$P$ value & & 0.355 & 0.006 & 0.134 & 0.211 & 0.009 & 0.114 \\
\hline $\begin{array}{l}\text { CYP2B6 } \\
\text { rs2279343 }\end{array}$ & $\begin{array}{c}A A \\
A G / G G\end{array}$ & $\begin{array}{c}1.00 \\
2.49 \\
(1.34-4.62)\end{array}$ & $\begin{array}{c}1.00 \\
16.03 \\
(2.03-126.5)\end{array}$ & $\begin{array}{c}1.00 \\
1.54 \\
(0.76-3.11)\end{array}$ & $\begin{array}{c}1.00 \\
1.94 \\
(1.11-5.02)\end{array}$ & $\begin{array}{c}1.00 \\
14.68 \\
(1.63-145.7)\end{array}$ & $\begin{array}{c}1.00 \\
1.16 \\
(0.68-3.78)\end{array}$ \\
\hline$P$ value & & 0.004 & 0.008 & 0.227 & 0.008 & 0.011 & 0.214 \\
\hline $\begin{array}{l}\text { CYP2CI9 } \\
\text { rs4244285 }\end{array}$ & $\begin{array}{c}\text { GG } \\
\text { GA/AA }\end{array}$ & $\begin{array}{c}1.00 \\
3.36 \\
(1.76-6.44)\end{array}$ & $\begin{array}{c}1.00 \\
12.61 \\
(1.60-49.5)\end{array}$ & $\begin{array}{c}1.00 \\
2.91 \\
(1.40-6.03)\end{array}$ & $\begin{array}{c}1.00 \\
3.29 \\
(1.94-4.59)\end{array}$ & $\begin{array}{c}1.00 \\
10.04 \\
(1.75-78.26)\end{array}$ & $\begin{array}{c}1.00 \\
2.12 \\
(1.19-4.32)\end{array}$ \\
\hline$P$ value & & 0.001 & 0.016 & 0.004 & 0.001 & 0.017 & 0.009 \\
\hline $\begin{array}{l}\text { NAT2 } \\
\text { rsI79993I }\end{array}$ & $\begin{array}{c}\text { GG } \\
\text { GA/AA }\end{array}$ & $\begin{array}{c}1.00 \\
1.78 \\
(0.98-3.21)\end{array}$ & $\begin{array}{c}1.00 \\
7.59 \\
(1.62-35.35)\end{array}$ & $\begin{array}{c}1.00 \\
1.39 \\
(0.67-2.85)\end{array}$ & $\begin{array}{c}1.00 \\
1.21 \\
(0.94-4.02)\end{array}$ & $\begin{array}{c}1.00 \\
11.02 \\
(2.79-50.36)\end{array}$ & $\begin{array}{c}1.00 \\
1.23 \\
(0.98-1.87)\end{array}$ \\
\hline$P$ value & & 0.776 & 0.001 & 0.369 & 0.054 & 0.005 & 0.421 \\
\hline $\begin{array}{l}\text { PONI } \\
\text { rs } 662\end{array}$ & $\begin{array}{c}A A \\
A G / G G\end{array}$ & $\begin{array}{c}1.00 \\
1.49 \\
(0.82-2.69)\end{array}$ & $\begin{array}{c}1.00 \\
9.58 \\
(1.21-75.6)\end{array}$ & $\begin{array}{c}1.00 \\
1.30 \\
(0.64-2.64)\end{array}$ & $\begin{array}{c}1.00 \\
1.67 \\
(0.69-3.04)\end{array}$ & $\begin{array}{c}1.00 \\
8.45 \\
(1.99-80.23)\end{array}$ & $\begin{array}{c}1.00 \\
1.65 \\
(0.67-2.14)\end{array}$ \\
\hline$P$ value & & 0.187 & 0.032 & 0.453 & 0.204 & 0.041 & 0.514 \\
\hline
\end{tabular}

Notes: OR and Adjusted OR analyzed by Binary logistic regression. *Adjusted for sex, age, smoking status, alcohol consumption, and exposure parameters. Abbreviations: $\mathrm{Cl}$, confidence interval; OR, odds ratio.

rs2279343, and CYP2C19 rs4244285) were associated with thyroid dysfunction. Compared with wildtype, in chemical workers, the OR $(95 \% \mathrm{CI})$ per one unit elevation of thyroid dysfunction for the CYP1A1 rs1048943 heterozygous/variant genotype was 18.14 (2.29-143.2); a similar result was found in the adjusted model (OR: 15.12, 95\% CI: $2.01-$ 134.6). As shown in Table 7, the CYP2B6 rs2279343 heterozygous/variant genotype showed a significant risk of thyroid dysfunction for all workers (OR: 2.49, 95\% CI: 1.34-4.62; AOR: 1.94, 95\% CI: 1.11-5.02) and for chemical workers (OR: 16.03, 95\% CI: 2.03-126.58 and AOR: 14.68, 95\% CI: 1.63-145.7). There was a significantly increased risk of thyroid dysfunction for those with the CYP2C19 rs4244285 heterozygous/variant genotype among all workers, chemical workers, and organic workers, as seen in the ORs $(3.36,95 \% \mathrm{CI}: 1.76-6.44 ; 12.61,95 \%$ CI: 1.60-49.5; and 2.91, 95\% CI: 1.40-6.03) and AORs
(3.29, 95\% CI: $1.94-4.59 ; 10.04,95 \%$ CI: 1.75-78.26; and 2.12, 95\% CI: 1.19-4.32, respectively). In addition, there was increased risk of abnormal thyroid function in chemical workers with the NAT2 rs1799931 (OR: 7.59, 95\% CI: 1.62-35.35; AOR: 11.02, 95\% CI: 2.79-50.36) and PON1 rs662 heterozygous/variant genotypes (OR: 9.58, 95\% CI: 1.21-75.63; AOR: 8.45, 95\% CI: 1.99-80.23).

\section{Discussion}

In recent decades, marked changes in agricultural practices have led to the extensive use of pesticides, including herbicides, insecticides, fungicides, nematicides, and rodenticides, which can be synthetic or natural. Active ingredients in synthetic pesticides may be toxic and cause adverse human health effects. Thyroid dysfunction has long been recognized as abnormal TSH, FT4, and FT3 concentrations, which can result from exposure to endocrine-disrupting pesticides. 
Susceptibility to these altered hormone concentrations partly depends on modifiable (smoking, alcohol intake, physical activity, underlying diseases, and exposure determinants) and non-modifiable factors (age, sex, family history, and ethnic or genetic background). In the present study, there were significant differences in age, sex, smoking status, and alcohol consumption between the chemical and organic worker groups, and these factors might have contributed to changes in thyroid hormone concentrations (Table 1). The effect of smoking status on thyroid function was supported by a population-based cohort study that investigated the relationship between smoking and altered thyroid hormone concentrations (FT4, TSH, and thyroid peroxidase antibody). ${ }^{19}$ Several possible underlying mechanisms were proposed, including 1) toxicant-induced stimulation of the thyroid gland, resulting in increased serum concentrations of thyroxine-binding globulin and T3, and decreased TSH, and 2) toxicants interfering with iodine transport. ${ }^{19}$ It has been noted that alcohol consumption can alter the HTP axis by downregulating the thyrotropin-releasing hormone receptor and subsequently reducing TSH secretion. ${ }^{20}$ For the effect of alcohol consumption on thyroid hormone levels have been described. Alcohol can induce direct cellular toxicity on thyroid cells thereby producing thyroid suppression and reducing thyroid volume. However, the mechanism of alcohol-induced reduction in TSH secretion to thyrotropinreleasing hormone (TRH) stimulation is important. One hypothesis for this phenomenon is a possible downregulation of the TRH receptors in the pituitary due to chronically high TRH concentrations. ${ }^{20}$

Details of pesticides use in this study were similar to those of a study of rice farmers ( $\mathrm{n}=182$, mean age $=54.3 \pm 9.5$ years) in Northern Thailand who stated that they commonly used insecticides $(\mathrm{n}=154,84.6 \%)$, herbicides $(\mathrm{n}=115$, $63.2 \%)$, and fungicides $(\mathrm{n}=13,7.1 \%)$ and had an increased prevalence of respiratory tract and muscle symptoms. ${ }^{3}$

The results suggest that the long-term use of pesticides by the chemical workers may have contributed to their significantly higher concentrations of TSH $(1.58 \mu \mathrm{IU} / \mathrm{mL})$ and FT3 $(0.34 \mathrm{ng} / \mathrm{dL})$ compared with the organic workers $(1.12 \mu \mathrm{IU} /$ $\mathrm{mL}$ and $0.31 \mathrm{ng} / \mathrm{dL}$, respectively) (Table 1). These results were comparable to those of a study in Iran (40 sprayers and 20 controls) that found significantly increased TSH (2.64 \pm 1.50 vs $1.33 \pm 0.83 \mathrm{mIU} / \mathrm{L}, P=0.007)$ and decreased T3 concentrations ( $1.12 \pm 0.52$ vs $1.55 \pm 0.57 \mathrm{nmol} / \mathrm{L}, P=0.018)$ in the sprayer group. No significant difference in T4 was found between the sprayers and controls $(9.29 \pm 2.12$ vs 10.85 $\pm 2.82 \mu \mathrm{g} / \mathrm{dL}, P=0.091) .{ }^{21}$ These findings together with the exposure data shown in Figure 1 indicate that changes in thyroid hormone concentrations may involve the effects of insecticides, herbicides, and/or fungicides on the molecular regulation of the HTP axis. These effects might be caused by the pesticides interfering with central regulation, iodine uptake, the synthesis/function of thyroid hormones, and the production capacity of thyroid hormone receptors. ${ }^{22} \mathrm{TSH}$ is the most sensitive biomarker of thyroid status. Hypothyroidism can clinically present as an increased TSH concentration along with low concentrations of other thyroid hormones, while hyperthyroidism presents as suppressed $\mathrm{TSH}$, with high concentrations of other thyroid hormones. ${ }^{23}$ Subclinical thyroid dysfunction can be detected as FT4, FT3, and $\mathrm{T} 3$ values that are within the reference ranges, with the TSH concentration being either high or low. ${ }^{15}$ We found that $3.7 \%$ and $8.3 \%$ of the chemical and organic workers had subclinical thyroid disease, and $38.4 \%$ and $42.4 \%$ had other type of thyroid dysfunction, respectively. Subclinical thyroid disease is more prevalent in the elderly and in women without difference in genetic factors. The more prevalence of subclinical thyroid disease in Table 1 was found compared to chemical workers, possibly due to more number of women. ${ }^{23}$ The Agricultural Health Study (AHS) was a prospective cohort study of licensed pesticide applicators (mainly farmers; 35 men, 150 women) in the USA that found evidence of hypothyroidism in private pesticide applicators. ${ }^{24}$ However, our results were not similar to the findings from a subset of the AHS participants, where a higher exposure to the insecticide Aldrin and the herbicide pendimethalin was positively associated with subclinical hypothyroidism. ${ }^{25}$ Thus, the interpretation of thyroid hormone disruption should take several issues into account, such as the level and duration of exposure, the genetic background of individual agricultural workers, and the ability of pesticides to have different effects in the different age groups and sex.

To our knowledge, this is the first study that has investigated a large number of SNPs in agricultural workers to assess the potential risk of thyroid dysfunction. The genetic analysis of enzymes involved in pesticide metabolism provides valuable information regarding individuals or populations that may have an increased health risk because of their polymorphism profile. Genetic variations of these genes can lead to pesticides forming highly toxic intermediates and ultimately damaging various molecular targets. Here, the genomic DNA of two groups of agricultural workers was analyzed to detect the frequencies of 15 metabolically related genes (CYP1A1, CYP1A2, CYP $3 A 5$, 
CYP2B6, CYP2C19, CYP2D6, CYP2E1, POR1, NAT1, NAT2, PON1, ABCB1, GSTP1, GSTT1, and GSTM1). The allele frequencies of all genes were in HardyWeinberg equilibrium. The mutant allele and null genotype frequencies were compared with those found in reports of Thai and other Asian populations. ${ }^{26-29}$ For example, the allele frequencies of CYP1A1 rs1048943 ( 0.21 for chemical workers and 0.31 for organic workers) and rs4646903 (0.49 for chemical workers and 0.42 for organic workers) found in this study were similar to those in another report of a Thai population (0.29 and 0.50, respectively). ${ }^{26}$ For two CYP1A2 SNPs, our findings were in agreement with the minor allele frequency found in a Korean population ( 0.35 for rs762551 and 0.25 for rs2069514). ${ }^{27}$ CYP2E1 is an enzyme that functions in the metabolic pathways of synthetic pesticides. Sangrajrang et al found MAFs of 0.14 for rs2031920/rs3813867 and 0.20 for rs $6413432,{ }^{28}$ which were similar to those found in the current study. In addition, the null GSTM1 genotype in a Thai population was previously determined to be $62.7 \%,{ }^{28}$ compared with $64-66 \%$ in our workers. PON1 gene polymorphism has been suggested as a critical risk factor for adverse health effects related to organophosphorus insecticides. The frequencies of PON1 mutant alleles (rs662, rs705379, and rs854560) in the two worker groups were in agreement with those previously reported for the Thai population. ${ }^{29}$

Another group of phase II enzymes includes $\mathrm{N}$-acetyltransferase 1 (NAT1) and 2 (NAT2), which are conjugating enzymes involved in the activation and inactivation reactions of many drugs and xenobiotics. Both genes exhibit genetic polymorphisms, with biochemical phenotypes ranging from slow to fast acetylators. ${ }^{30}$ The frequency distribution of the major NAT1 alleles (rs15561 and rs1057126) found in this study and in a report by Kukongviriyapan et $\mathrm{al}^{31}$ shows a similar pattern to that in other Asian populations. ${ }^{32}$ In the current study, three different allelic variants of the NAT2 gene were identified (rs1041983, rs1799931, and rs1799930) (Table 3), and the allele frequencies were in agreement with previous reports of the $\mathrm{Thai}^{33}$ and Chinese $^{34}$ populations. The current study is the first to investigate the relationships between genetic variation in the $A B C B 1$ gene, pesticide exposure, and thyroid dysfunction. This gene is located on chromosome 7q21.12 and encodes a 1280 amino acid protein. There was linkage disequilibrium between G2677T (rs2032582) located on exon 21, C3435T (rs1045642) on exon 26, and C1236T (rs1128503) on exon 12. A functional SNP, C3435T (rs1045642), was found to have a more than two-fold lower duodenal ABCB1 expression level for the homozygous $\mathrm{T}$ allele compared with the $\mathrm{CC}$ allele. ${ }^{35}$ These data suggest that interpersonal variability in this transporter may result in alterations in the rate and extent of toxicant oral absorption. The allele frequencies of $A B C B 1$ rs1045642 and rs1128503 in the chemical and organic workers were similar to those found in previous studies in Asian populations. ${ }^{36,37}$ The $P O R$ gene encodes a flavoprotein that is necessary for the electron transport of cytochrome P450 enzymes, such as CYP3A4 and CYP3A5. Individuals with $P O R$ mutations may have altered drug or chemical metabolism. ${ }^{38}$ Two SNPs of POR (rs1057868 and rs1135612) were genotyped in all workers. POR1 rs1057868 (POR*28) is the most common mutation, with high frequencies ranging from $19 \%$ to $37 \%$ across all major ethnicities, ${ }^{38}$ which is comparable to our finding.

When individuals with genetic polymorphisms of metabolizing enzymes and transporters are exposed to pesticides, such as in an intensive agricultural setting, it might result in altered thyroid function arising from endocrine disruption. The frequencies of individual variants were compared among individuals exhibiting different thyroid hormone concentrations. Pesticide metabolism mainly involves the various isoforms of cytochrome P450s, which have different expression levels and enzyme activities. The thyroid hormone concentrations in workers with CYP polymorphisms are shown in Tables 4 and 5. The chemical workers with heterozygous/homozygous variants of cytochrome P450s, such as CYP1A1, 3A5, 2B6, 2D6, 2E1, and $2 C 19$, showed significant differences in TSH, FT4, and FT3 concentrations compared with the organic workers (all $P<0.05)$. These levels were within the reference ranges, indicating no marked effect of these genetic variations. However, if the chemical workers have long-term exposure, they might be susceptible to impaired thyroid function because the $C Y P$ variants might lead to more toxic intermediates that could induce oxidative stress and inflammation. Our results were in agreement with a previous study that exposed rats to fipronil sulfone and found increased CYP2B- and CYP3A-dependent biotransformation, which induced changes in thyroid metabolism. ${ }^{39}$

The acetylator status for NAT1 and NAT2 was also determined by genotyping. Owing to occupational pesticide exposure, acetylation by these enzymes is regarded as a detoxification step. Interestingly, there was an association between the SNPs of NAT1 and NAT2 and the TSH, FT4, and FT3 serum concentrations in both worker groups (Table 5). Our findings indicate that polymorphisms in human NAT 
result in the production of NAT proteins with variable enzyme activity or stability, leading to possible pesticideinduced toxicity and an increased risk of thyroid dysfunction. $\mathrm{Xu}$ et al provided toxicokinetic evidence that the pesticide dithiocarbamate is linked to alterations in NAT1 enzyme function. ${ }^{40}$ GSTs are another important group of phase II enzymes, and individuals with polymorphic GST genes possibly have impaired activity to metabolically eliminate toxic intermediate compounds, and therefore might be at increased risk of abnormal thyroid function. In this study, chemical workers with GSTM1, GSTT1, or GSTP1 polymorphisms showed significant differences in serum TSH and FT3 concentrations compared with organic workers who also had these genetic alterations. Evidence of occupational exposure with pesticides and role of NATs or GSTs mostly related to thyroid cancer contrast to limited data involved in thyroid hormones. The possible relationship between GSTM1, GSTT1, GSTP1, and NAT2 polymorphisms and an increased risk of thyroid cancer was previously evaluated in 176 thyroid cancer patients and 167 healthy controls. ${ }^{41}$

Variability in OP metabolism can also arise as a result of specific CYP and PON1 isoforms. OP oxons are substrates of PON1, and their metabolism results in detoxification. ${ }^{42}$ Polymorphisms of the PON1 gene are responsible for variations in the expression and catalytic activity of the PON1 enzyme, leading to differential sensitivities to OP-induced toxicity. Our results showed significant differences in the TSH and FT3 concentrations of chemical and organic workers who had the heterozygous/homozygous variants of PON1 rs662, rs705379, and a combination of at least two PON1 SNPs. A previous study in male floriculture workers that investigated biomarkers of exposure (dialkylphosphates; DAPs), susceptibility (PON1 polymorphisms and activity), and effect (thyroid hormone concentrations) found evidence for relationship between these biomarkers. Workers with the PON1 rs662 genotype showed a greater increase in TSH concentration for each logarithmic unit increment in DAP compared with those who had the wild-type or heterozygous genotype (percentage of variation $59 \%, 23 \%$, and $14 \%$, respectively). ${ }^{43}$

Our findings indicate that adverse health effects related to thyroid dysfunction in the investigated high-risk population were partly determined by genetic variations of enzymes and transporters. Various molecular mechanisms may support an interaction between pesticides, polymorphisms, thyroid hormones, and thyroid hormone receptors through oxidative stress and cellular damage within the thyroid gland. The mechanisms by which pesticide exposure is thought to alter thyroid hormone concentrations were identified by analyzing the data with STITCH 4.0, which is a tool for identifying chemical interactions (Figure 2). Exposure to pesticides (eg, chlorpyrifos, methyl parathion, carbofuran, carbosulfan, cypermethrin, abamectin, profenofos, glyphosate, paraquat dichloride, 2,4-D sodium salt, diuron, amethrin, acetochlor, and cypermethrin), the investigated SNPs (GSTP1, GSTM1, GSTT1, PON1, NAT1, NAT2, CYP1A1, CYP1A2, CYP3A5, CYP2B6, CYP2C19, CYP2D6, CYP2E1, cytochrome P450 oxidoreductase, and ATP-binding cassette sub-family B), and thyroid hormone concentrations were found to be closely related and possibly linked to thyroid function interference. Oxidative stress markers (hydrogen peroxide, malondialdehyde, and 4-hydroxynonenal) also indicate oxidative stress as an underlying pathway related to pesticide exposure and endocrine disruption.

This study is the first to explore whether genetic polymorphisms in enzymes and transporters among agricultural workers are associated with pesticide-induced alterations in thyroid hormones. In conjunction with GST polymorphisms, the various pesticide mixtures and their ability to induce CYP and/or inhibit NAT can lead to an increased risk of thyroid dysfunction. However, some study limitations should be noted. First, although the number of workers met the requirement for statistical analysis, the sample size was too small to fully investigate the clinical manifestations of thyroid disorders in each polymorphism group. Second, modifiable risk factors, such as environmental/lifestyle factors and occupational exposure, as well as clinical evidence related to endocrine function, were not considered owing to the lack of detailed background information of the participants. These factors could have influenced the study outcomes. Third, our results cannot be extended to other populations without considering ethnicity and health status. Last, the phenotype for a given genotype may have varied between the worker groups because the phenotype can depend on environmental factors, such as educational level, nutritional status, preventive health care, and exposure to various toxicants in the home or elsewhere.

\section{Conclusions}

This study contributes to our understanding of the distribution and frequencies of alleles of xenobiotic-metabolizing enzymes and transporters. These polymorphisms may, in part, explain why individuals can differ in their response to pesticide exposure. These findings support a possible role of pesticide exposure in adverse thyroid function and should focus public and environmental health concerns regarding the occupational risk associated with pesticide use. The 


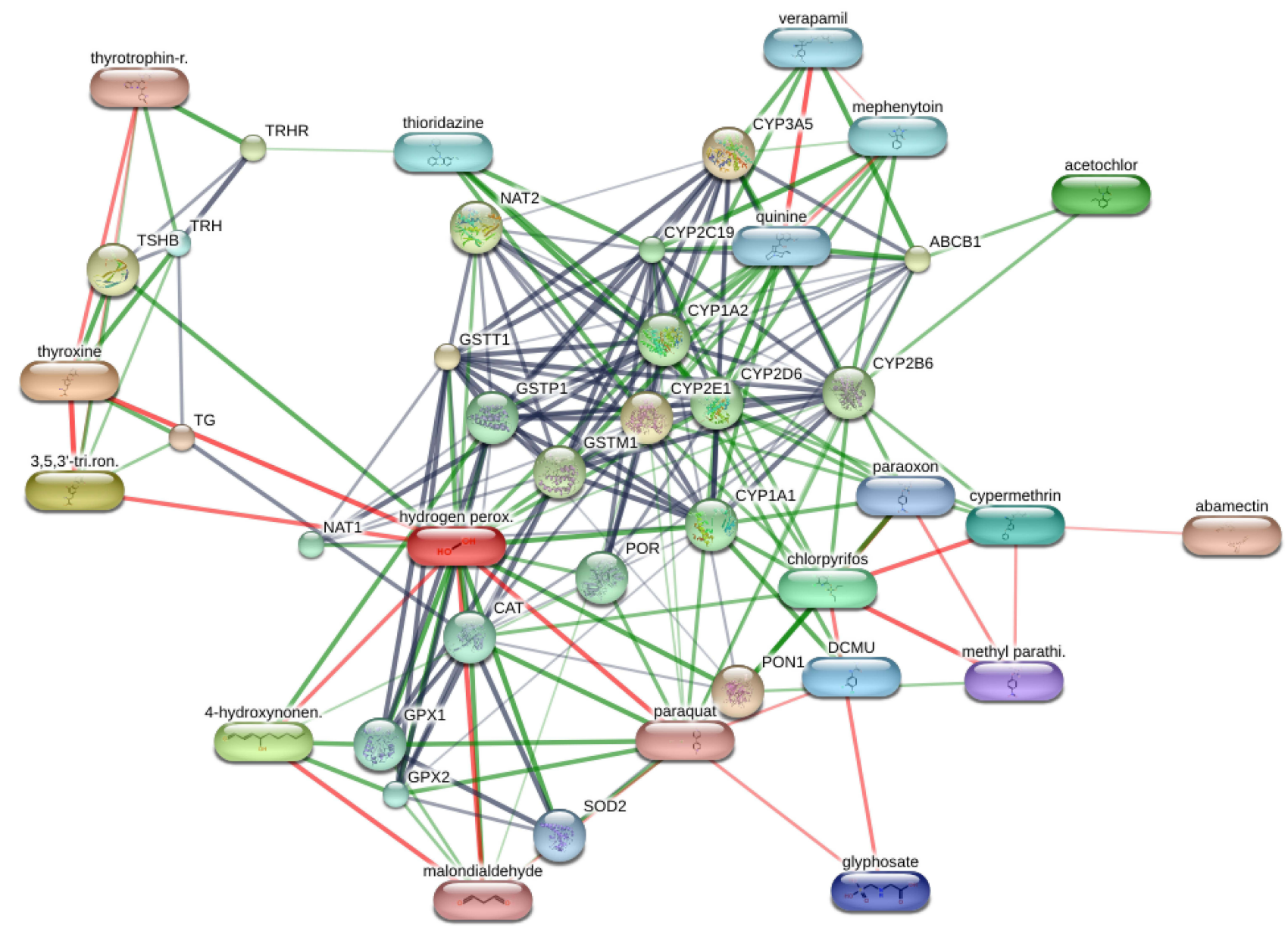

Figure 2 Proposed mechanisms by which pesticide exposure and xenobiotic-metabolizing enzymes/transporters interact to alter thyroid hormone concentrations. (Stronger associations are represented by thicker lines. Protein-protein interactions are shown in gray, chemical-protein interactions in green, and interactions between chemicals in red).

issues raised in this study are of scientific interest and have policy implications.

\section{Abbreviation}

3,5,3'-tri.ron, 3,5,3'-triiodothyronine; 95\% CI, 95\% confidence interval; ABCB1, ATP Binding Cassette Subfamily B Member 1; AORs, adjusted ORs; BMI, body mass index; CAT, catalase; CPF, chlorpyrifos; CYP, Cytochrome P450; DCMU, 3-(3,4-Dichlorophenyl)-1,1-dimethylurea; EDCs, endocrine-disrupting chemicals; FT3, free triiodothyronine; FT4, free thyroxine; HTP-axis, hypothalamic-pituitary-thyroid axis; HWE, Hardy-Weinberg equilibrium; GSTM1, Glutathione S-Transferase $\mathrm{Mu} \mathrm{1;}$ GSTP1, Glutathione S-transferase Pi 1; GSTT1, Glutathione S-transferase theta 1; MAFs, minor allele frequencies; NAT1, N-acetyltransferase 1; NAT2, N-acetyltransferase 2; ORs, odd ratios; PON1, paraoxonase 1; POR, P450 (cytochrome) oxidoreductase; PCRRFLP, PCR-restriction fragment length polymorphism; ROS, reactive oxygen species; SNPs, single nucleotide polymorphisms; TG, thyroglobulin; TRH, thyrotropin-releasing hormone; TRHR, thyrotropin-releasing hormone receptor; TSH, thyroid-stimulating hormone; TSHB, Thyroid Stimulating Hormone Subunit Beta.

\section{Acknowledgments}

We thank all of the workers who participated in this study. We also thank the participating hospital staff for their assistance in participant recruitment and data collection.

\section{Funding}

Funding for this project was provided by the Fogarty International Center and the National Institute of Environmental Health Sciences of the National Institutes of Health, as well as the National Institute for Occupational Safety and Health of the US Centers for Disease Control and Prevention under the Global 
Environmental and Occupational Health program (U01TW010091 and U2RTW010088).

\section{Disclosure}

The authors report no conflicts of interest associated with this work.

\section{References}

1. Office of Agricultural Regulation, Department of Agriculture, Thailand. Statistical data of hazardous chemicals control group, 2011-2017. Available from: https://www.doa.go.th /ard/?page_id= 386. Accessed February 10, 2020.

2. Kongtip P, Nankongnab N, Mahaboonpeeti R, et al. Differences among Thai agricultural workers' health, working conditions, and pesticide use by farm type. Ann Work Expo Health. 2018;62(2):167-181.

3. Sapbamrer R, Nata S. Health symptoms related to pesticide exposure and agricultural tasks among rice farmers from Northern Thailand. Environ Health Prev Med. 2014;19(1):12-20.

4. Teodoro M, Briguglio G, Fenga C, et al. Genetic polymorphisms as determinants of pesticide toxicity: Recent advances. Toxicol Rep. 2019;6:564-570. doi:10.1016/j.toxrep.2019.06.004

5. Sirivarasai J, Kaojarern S, Yoovathaworn K, et al. Paraoxonase (PON1) polymorphism and activity as the determinants of sensitivity to organophosphates in human subjects. Chem Biol Interact. 2007;168(3):184-192.

6. Mnif W, Hassine AI, Bouaziz A, et al. Effect of endocrine disruptor pesticides: a review. Int $J$ Environ Res Public Health. 2011;8 (6):2265-2303.

7. Chebab S, Mekircha F, Leghouchi E. Potential protective effect of Pistacia lentiscus oil against chlorpyrifos-induced hormonal changes and oxidative damage in ovaries and thyroid of female rats. Biomed Pharmacother. 2017;96:1310-1316.

8. Eaton DL, Daroff RB, Autrup H, et al. Review of the toxicology of chlorpyrifos with an emphasis on human exposure and neurodevelopment. crit Rev Toxicol. 2008;38(Suppl 2):1-125.

9. Beckman Coulter, Inc. Access TSH (3rd IS) Instructions for Use. Available from: https://www.beckmancoulter.com/en/products/immu noassay/access-tsh-3rd-is. Accessed July 28, 2019.

10. Beckman Coulter, Inc. Access Free T3 Instructions for Use. Available from: https://www.beckmancoulter.com/products/part/A13422\#/docu ments. Accessed July 28, 2019.

11. Beckman Coulter, Inc. Access free T4 Instructions for Use. Available from: https://www.beckmancoulter.com/products/part/33880\#. Accessed July 28, 2019.

12. Arici M, Özhan G. The genetic profiles of CYP1A1, CYP1A2 and CYP2E1 enzymes as susceptibility factor in xenobiotic toxicity in Turkish population. Saudi Pharm J. 2017;25(2):294-297.

13. Liu S, Park JY, Schantz SP, et al. Elucidation of CYP2E1 5' regulatory RsaI/Pstl allelic variants and their role in risk for oral cancer. Oral Oncol. 2001;37(5):437-445.

14. Yassine IA, Kobeissi L, Jabbour ME, et al. N-Acetyltransferase 1 (NAT1) Genotype: a risk factor for urinary bladder cancer in a Lebanese population. J Oncol. 2012;2012:512976.

15. Koulouri O, Gurnell M. How to interpret thyroid function tests. Clin Med. 2013;13(3):282-286.

16. Cooper DS, Biondi B. Subclinical thyroid disease. Lancet. 2012;379 (9821):1142-1154.

17. Rugge B, Balshem H, Sehgal R, et al. Screening and treatment of subclinical hypothyroidism or hyperthyroidism. Rockville (MD): Agency for Healthcare Research and Quality (US); 2011 Oct. (Comparative Effectiveness Reviews, No. 24.) Introduction. Available from: https://www.ncbi.nlm.nih.gov/books/NBK83492/.
18. Kuhn M, Szklarczyk D, Pletscher-Frankild S, et al. STITCH 4: integration of protein-chemical interactions with user data. Nucleic Acids Res. 2014;42(D1):D401-D407.

19. Kim SJ, Kim MJ, Yoon SG, et al. Impact of smoking on thyroid gland: dose-related effect of urinary cotinine levels on thyroid function and thyroid autoimmunity. Sci Rep. 2019;9(1):4213.

20. Balhara YP, Deb KS. Impact of alcohol use on thyroid function. Indian J Endocrinol Metab. 2013;17(4):580-587.

21. Farokh F, Taravati A. Pesticide exposure and thyroid function in adult male sprayers. Int J Med Invest. 2014;3(4):127-132.

22. Leemans M, Couderq S, Demeneix B, et al. Pesticides with potential thyroid hormone-disrupting effects: a review of recent data. Front Endocrinol (Lausanne). 2019;10:743.

23. Jameson J. Chapter 335 disorders of the thyroid gland. In: Fauci AS, Braunwald E, Kasper DL, et al., editors. Harrison's Principles of Internal Medicine. New York: The McGraw-Hill Companies, Inc; 2008:2224-2246.

24. Shrestha S, Parks CG, Goldner WS, et al. Pesticide use and incident hypothyroidism in pesticide applicators in the agricultural health study. Environ Health Perspect. 2018;126(9):97008.

25. Lerro CC, Beane Freeman LE, DellaValle CT, et al. Occupational pesticide exposure and subclinical hypothyroidism among male pesticide applicators. Occup Environ Med. 2018;75(2):79-89.

26. Wongpratate M, Ishida W, Phuthong S, et al. Genetic polymorphisms of the human cytochrome P450 1A1 (CYP1A1) and cervical cancer susceptibility among northeast Thai women. Asian Pac J Cancer Prev. 2020;21(1):243-248.

27. Jung KS, Hong KW, Jo HY, et al. KRGDB: the large-scale variant database of 1722 Koreans based on whole genome sequencing. Database (Oxford). 2020;2020:baz146.

28. Sangrajrang S, Jedpiyawongse A, Srivatanakul P. Genetic polymorphisms of CYP2E1 and GSTM1 in a Thai population. Asian Pac J Cancer Prev. 2006;7(3):415-419.

29. Phuntuwate W, Suthisisang C, Koanantakul B, Mackness MI, Mackness B. Paraoxonase 1 status in the Thai population. J Hum Genet. 2005;50(6):293-300.

30. Hein DW, Doll MA, Fretland AJ, et al. Molecular genetics and epidemiology of the NAT1 and NAT2 acetylation polymorphisms. Cancer Epidemiol Biomarkers Prev. 2000;9(1):29-42.

31. Kukongviriyapan V, Prawan A, Warasiha B, et al. Polymorphism of $\mathrm{N}$-acetyltransferase 1 and correlation between genotype and phenotype in a Thai population. Eur J Clin Pharmacol. 2003;59 (4):277-281.

32. Zhangwei X, Jianming X, Qiao M, et al. N-Acetyltransferase-1 gene polymorphisms and correlation between genotype and its activity in a central Chinese Han population. Clin Chim Acta. 2006;371(12):85-91.

33. Kukongviriyapan V, Prawan A, Tassaneyakul W, et al. Arylamine $\mathrm{N}$-acetyltransferase-2 genotypes in the Thai population. $\mathrm{Br} J$ Clin Pharmacol. 2003;55(3):278-281.

34. Huang Z, Yuan L, Jiang Z, et al. Associations of polymorphisms in NAT2 gene with risk and metastasis of osteosarcoma in young Chinese population. Onco Targets Ther. 2015;8:2675-2680.

35. Hoffmeyer S, Burk O, von Richter O, et al. Functional polymorphisms of the human multidrug-resistance gene: multiple sequence variations and correlation of one allele with P-glycoprotein expression and activity in vivo. Proc Natl Acad Sci USA. 2000;97:3473-3478.

36. Balram C, Sharma A, Sivathasan C, et al. Frequency of C3435T single nucleotide MDR1 genetic polymorphism in an Asian population: phenotypic-genotypic correlates. Br J Clin Pharmacol. 2003;56 (1):78-83.

37. Fung KL, Gottesman MM. A synonymous polymorphism in a common MDR1 (ABCB1) haplotype shapes protein function. Biochim Biophys Acta. 2009;1794:860-871. 
38. Zanger UM, Schwab M. Cytochrome P450 enzymes in drug metabolism: regulation of gene expression, enzyme activities, and impact of genetic variation. Pharmacol Ther. 2013;138(1):103-141.

39. Roques BB, Lacroix MZ, Puel S, et al. CYP450-dependent biotransformation of the insecticide fipronil into fipronil sulfone can mediate fipronil-induced thyroid disruption in rats. Toxicol Sci. 2012;127 (1):29-41.

40. Xu X, Mathieu C, Berthelet J, et al. Human arylamine N-acetyltransferase 1 is inhibited by the dithiocarbamate pesticide thiram. Mol Pharmacol. 2017;92(3):358-365.
41. Hernández A, Xamena N, Surrallés J, et al. Role of GST and NAT2 polymorphisms in thyroid cancer. J Endocrinol Invest. 2008;31 (11):1025-1031.

42. Furlong CE, Cole TB, Jarvik GP, et al. Pharmacogenomic considerations of the paraoxonase polymorphisms. Pharmacogenomics. 2002;3 (3):341-348

43. Lacasaña M, López-Flores I, Rodríguez-Barranco $M$, et al. Interaction between organophosphate pesticide exposure and PON1 activity on thyroid function. Toxicol Appl Pharmacol. 2010;249 (1):16-24.

\section{Publish your work in this journal}

Risk Management and Healthcare Policy is an international, peerreviewed, open access journal focusing on all aspects of public health, policy, and preventative measures to promote good health and improve morbidity and mortality in the population. The journal welcomes submitted papers covering original research, basic science, clinical \& epidemiological studies, reviews and evaluations, guidelines, expert opinion and commentary, case reports and extended reports. The manuscript management system is completely online and includes a very quick and fair peer-review system, which is all easy to use. Visit http://www.dovepress.com/testimonials.php to read real quotes from published authors.

Submit your manuscript here: https://www.dovepress.com/risk-management-and-healthcare-policy-journal 